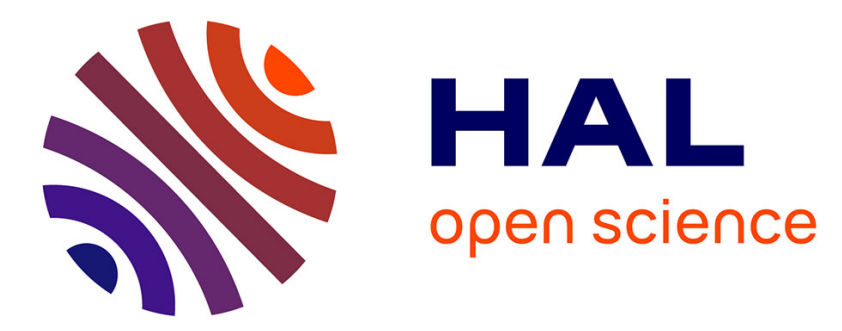

\title{
On the low-Reynolds-number flow about two rotating circular cylinders
}

\author{
Y. Ueda, Antoine Sellier, T. Kida, M. Nakanishi
}

\section{To cite this version:}

Y. Ueda, Antoine Sellier, T. Kida, M. Nakanishi. On the low-Reynolds-number flow about two rotating circular cylinders. Journal of Fluid Mechanics, 2003, november (495), pp.255-281. $10.1017 / \mathrm{s} 002211200300627 \mathrm{x}$. hal-01024921

\section{HAL Id: hal-01024921}

https://hal-polytechnique.archives-ouvertes.fr/hal-01024921

Submitted on 27 Aug 2014

HAL is a multi-disciplinary open access archive for the deposit and dissemination of scientific research documents, whether they are published or not. The documents may come from teaching and research institutions in France or abroad, or from public or private research centers.
L'archive ouverte pluridisciplinaire HAL, est destinée au dépôt et à la diffusion de documents scientifiques de niveau recherche, publiés ou non, émanant des établissements d'enseignement et de recherche français ou étrangers, des laboratoires publics ou privés. 


\title{
On the low-Reynolds-number flow about two rotating circular cylinders
}

\author{
By Y. UEDA ${ }^{1} \uparrow, A$ A SELLIER ${ }^{2}$, T. KIDA ${ }^{1}$ AND M. NAKANISHI \\ ${ }^{1}$ Department of Energy Systems Engineering, Osaka Prefecture University, Sakai, Osaka 599-8531, Japan \\ ${ }^{2}$ LadHyX, Ecole Polytechnique, 91128 Palaiseau Cédex, France \\ ${ }^{3}$ Hitachi, Ltd., Mechanical Engineering Research Laboratory, 502 Kandatsu, Tsuchiura, Ibaraki \\ 300-0013, Japan
}

(Received 27 January 2003 and in revised form 10 July 2003)

The viscous and steady flow about two distinct parallel infinite rotating circular cylinders is theoretically investigated. Because any inner steady Stokes flow is not quiescent far from the cylinders, a strictly steady analysis requires matching an inner steady Stokes approximation with an outer solution of the steady Navier-Stokes equations. However, except for the case of identical cylinders of equal angular velocities, it is impossible to determine this outer solution. In the same spirit as Nakanishi et al. (1997) and Ueda et al. (2001), the present work therefore first addresses the unsteady viscous flow induced by cylinders impulsively set into both steady rotation and translation $\boldsymbol{W}$. Using integral representations of the stream function and the vorticity, the resulting long-time flow is approximated in the limit of large viscosity. Letting time tend to infinity for $\boldsymbol{W}$ non-zero extends Lee \& Leal (1986) and agrees with Watson (1996), whereas the required steady flow is obtained by making $\boldsymbol{W}$ vanish before letting time go to infinity. At the obtained leading-order approximation, the 'lift' and 'drag' forces on each cylinder (parallel and normal to the line of centres) are respectively zero and independent of the Reynolds number. The drag experienced by each body is plotted versus the gap between the cylinders for several values of the rotation, both for identical and non-identical cylinders.

\section{Introduction}

We consider two parallel infinite circular cylinders embedded, as sketched in figure 1(a), in an unbounded and Newtonian fluid of uniform kinematic viscosity $\nu$ and density $\rho$.

Let each cylinder $C_{n}$, of radius $a_{n}$, start rotating about its axis, parallel to $\boldsymbol{e}_{z}$, with constant angular velocity $\Omega_{n} \boldsymbol{e}_{z}$. As the problem necessarily requires solving the full unsteady Navier-Stokes equations, the numerical determination of the induced time-dependent and two-dimensional flow appears to be non-trivial. However, at least for a sufficiently viscous flow, one expects to quickly reach a steady regime, quiescent at infinity. Furthermore, it remains of prime interest theoretically to approximate the main properties (for instance, the force per unit length experienced by each cylinder) of such a steady state. Therefore, several authors have addressed this issue, starting 

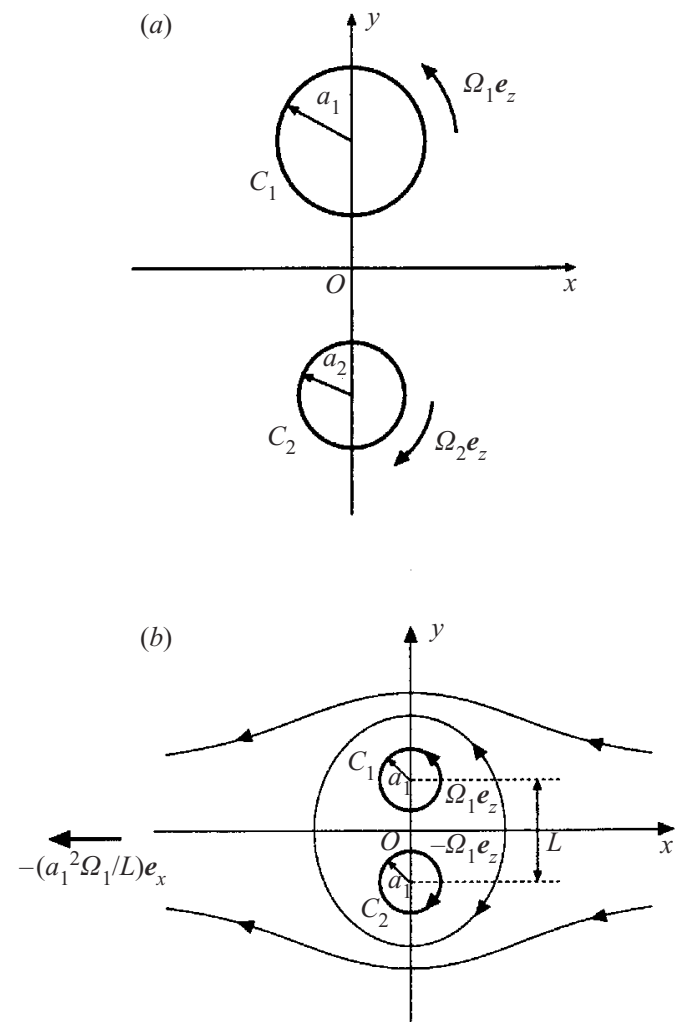

FIGURE 1. (a) Two co-rotating circular cylinders $\left(\Omega_{1} \Omega_{2}>0\right)$ immersed in an unbounded viscous fluid. (b) Jeffery's simple illustrating example of identical circular cylinders $\left(a_{2}=a_{1}\right)$ of opposite angular velocities $\left(\Omega_{2}=-\Omega_{1}<0\right)$. A few streamlines are drawn.

with the pioneering work of Jeffery (1922). As first pointed out in that paper, it is in general not possible to find a steady Stokes flow that simultaneously fulfils the no-slip boundary condition on each cylinder and vanishes far from the cluster: this constitutes the so-called Jeffery paradox. As a simple illustrative example, Jeffery (1922) found that the steady Stokes flow around two equal $\left(a_{2}=a_{1}\right)$ cylinders of centre-to-centre distance $L$ and opposite angular velocities $\left(\Omega_{2}=-\Omega_{1}\right)$ exhibits at infinity the uniform velocity $-\left[a_{1}^{2} \Omega_{1} / L\right] \boldsymbol{e}_{x}$, normal to the line of centres (see figure $1 b$ ). The associated streamline pattern, available in Dorrepaal, O'Neill \& Ranger (1984) or Elliott, Ingham \& El Bashir (1995), is symmetric about the $x$ - and $y$-axes and exhibits two stagnation points on the $x$-axis. In addition, the flow yields zero net force and torque on the cluster, a finite force and torque on each cylinder but an infinite fluid kinetic energy. As detailed by Smith (1991) and incidentally first noticed by Umemura (1982), the far-field behaviour of Jeffery's Stokes flow for a general parameter setting $\left(a_{1}, a_{2}, \Omega_{1}, \Omega_{2}\right)$ consists of a uniform stream plus a solid-body rotation which vanishes with $a_{1}^{2} \Omega_{1}+a_{2}^{2} \Omega_{2}$. As a consequence, the two-cylinder cluster is seen to systematically experience zero net force and torque. By using a boundary element method, Elliott et al. (1995) computed the associated streamline and vorticity patterns both for identical cylinders (with $\Omega_{1}=-\Omega_{2}$ or $\Omega_{1}=-8 \Omega_{2}$ ) and non-identical cylinders (with $a_{2}=2 a_{1}$ and $\Omega_{1}=-4 \Omega_{2}$ ). In the same spirit as Kaplun \& Lagerstrom (1957), Smith (1991) furthermore considered Jeffery's flow as an inner solution and proposed an outer 
stream function, $\psi$, solution to the (steady) Navier-Stokes equations, of the form $\psi=A r^{2}+B \log r, \quad r=|\boldsymbol{x}|$. Unfortunately, as observed by Watson (1995), Smith's treatment is subject to the following problems:

(i) The cluster always experiences zero net force and torque. This remark also applies to Elliott et al. (1995).

(ii) If $a_{1}^{2} \Omega_{1}+a_{2}^{2} \Omega_{2} \neq 0$ there is a solid-body rotation at infinity. This strongly contrasts with the case of a single rotating circular cylinder for which the far-field steady flow is produced by a line vortex at the axis of the cylinder.

(iii) Since it fulfils the Stokes equations, Smith's outer stream function yields, through the $B \log r$ term, a multiple-valued pressure.

As alluded to in Jeffery (1920) and apparently independently recovered by Umemura (1982), the Jeffery (1922) solution, which adopts the form of separation of variables in bipolar coordinates, discards other Stokes solutions which would allow for non-zero net force and torque on the cluster. Re-introducing these forgotten terms and requiring a zero far-field rotation, Watson (1995) found that, at leading-order of approximation, the force on each cylinder is parallel to $\boldsymbol{e}_{x}$ but the required steady flow was found only about two identical cylinders of equal rotation rate $\left(a_{1}=a_{2}, \Omega_{1}=\Omega_{2}\right)$. For other general parameter settings $\left(a_{1} \neq a_{2}\right.$ or $\left.\Omega_{1} \neq \Omega_{2}\right)$ the inner stream function $\psi$ behaves as $(C \log r+D) y$ as $r \rightarrow \infty$ with $4 \pi v \rho C$ the net $x$-component force on the cluster. It was then not possible to obtain the outer stream function solution to the full (steady) Navier-Stokes equations that matches such an inner far-field behaviour (and thereby determines $C$ ).

Since obtaining the outer flow in the refined treatment of Watson (1995) appears to be as cumbersome as numerically solving the time-dependent problem, another approach is required. The problem of a steady flow, no longer quiescent but of uniform velocity $\boldsymbol{W}$ at infinity, past one or several motionless or rotating cylinders is much less complicated and for this reason it has attracted much attention in the past few decades. In this respect, one should first mention the pioneering work by Stokes (1850) who considered the case of an oscillating circular cylinder, under the Stokes approximations in the whole fluid domain, and found his famous difficulty when looking at the zero-frequency limit. The viscous steady flow past one or two fixed circular cylinders of typical size $a$ was further approximated, as $a|\boldsymbol{W}| / v$ vanishes, by Tomotika \& Aoi (1950, 1951), Fujikawa (1956, 1957) and Kuwabara (1957), by using the Oseen approximation in the whole fluid domain. Within this 'entire' Oseen framework, Yano \& Kieda (1980) also proposed a discrete singularity method valid for a fixed and arbitrarily shaped cluster or single body. The no-slip boundary condition was adequately addressed by combining inner Stokes and outer Oseen approximations via the method of matched asymptotic expansions by Kaplun (1957) and Proudman \& Pearson (1957) for a circular cylinder, by Shintani, Umemura \& Takano (1983) for an inclined elliptic cylinder, by Umemura (1982) for two equal circular cylinders and by Lee \& Leal (1986) for multiple cylindrical bodies of arbitrary cross-sectional shapes. Note that Lee \& Leal (1986) resorted to Stokes and Oseen integral representations in order to deal with fixed but arbitrarily shaped bodies. Finally, both experimental and numerical results are available for the viscous flow past a single rotating circular cylinder (see Badr et al. 1990 and references cited therein) but Watson (1996) apparently provides the sole theoretical analysis of the steady viscous flow past two rotating circular cylinders. By constructing the outer Oseen approximation and matching it with the general Stokes inner solution, Watson (1996) asymptotically obtained the required steady flow for any setting $\left(a_{1}, a_{2}, \Omega_{1}, \Omega_{2}\right)$ and an arbitrary orientation of $\boldsymbol{W}$. However, the net force on the two-cylinder cluster 
is seen to vanish as $|\boldsymbol{W}|$ tends to zero and this prevents us from reaching the required steady regime, quiescent at infinity, by letting the outer stream tend to zero in Watson's solution.

Since any steady analysis fails in providing the expected long-time steady flow about two rotating cylinders which is quiescent at infinity, it becomes necessary to approximate the induced unsteady flow in the limit of vanishing Reynolds number. This challenging task has been performed for a single circular cylinder impulsively set into translation by Nakanishi, Kida \& Nakajima (1997) or into combined translation and rotation by Ueda et al. (2001). At any finite time, the unsteady flow obtained was found to exponentially decay far from the cylinder and the long-time regime, for the general case of the rotating and translating cylinder, has been recently carefully investigated by Kida \& Ueda (2002). Implementing a vortex method, Nakanishi \& Kida (1999) demonstrated that the outer unsteady Oseen approximation used in these theoretical investigations also holds for the flow about two identical cylinders with opposite angular velocities (as in figure $1 b$ ). These results strongly suggest analysing by a similar procedure the unsteady flow induced by two circular cylinders when impulsively set into both rotation and translation. The required steady flow, quiescent at infinity, is then expected to be reached by letting time tend to infinity and each cylinder translation velocity vanish. The present work carries out such an analysis.

The paper is organized as follows. Section 2 states the governing unsteady boundaryvalue problem for impulsively translating and rotating circular cylinders and the key integral representations of the Laplace transforms of the induced stream and vorticity functions. The long-time behaviour of the flow is obtained for a vanishing Reynolds number in $\S 3$ by applying the method of matched asymptotic expansions. The motivating case of a two-cylinder cluster is analytically solved in $\S 4$. As previously observed by Kida \& Ueda (2002) for one translating and rotating cylinder, two different steady long-time regimes are then obtained depending on whether one first lets time tend to infinity before the translation velocity vanishes or one first stops the translation velocity before letting time go to infinity. Each associated flow is discussed and the leading-order approximation of the forces on the rotating cylinders is given. Finally, the drag force experienced by each cylinder is plotted versus the gap between them for different parameter settings $\left(a_{1}, a_{2}, \Omega_{1}, \Omega_{2}\right)$.

\section{Governing equations and relevant integral representations}

As previously explained, let us assume that at initial time $t=0$ each cylinder $C_{n}$, of radius $a_{n}$ abruptly starts rotating at the constant angular velocity $\Omega_{n} \boldsymbol{e}_{z}$ and translating at the constant velocity $-\boldsymbol{W}$ normal to $\boldsymbol{e}_{z}$. For convenience, we look at the induced unsteady fluid motion relative to the plane $\left(O, \boldsymbol{e}_{x}, \boldsymbol{e}_{y}\right)$ attached to the two-cylinder cluster (see figure 2).

In this plane the boundary of $C_{n}$ becomes a steady circle $\mathscr{C}_{n}$ with centre $O_{n}$ and we shall denote $\boldsymbol{O} \boldsymbol{O}_{n}=(-1)^{n+1} h_{n} \boldsymbol{e}_{y}$ with $h_{n}>0$ and $\boldsymbol{O}_{2} \boldsymbol{O}_{1}=\left(a_{1}+a_{2}+h\right) \boldsymbol{e}_{y}$ where $h=h_{1}+h_{2}-\left(a_{1}+a_{2}\right)>0$ designates the gap between the cylinders.

Lengths, vorticity, time, velocities and pressure are normalized with characteristic scales $a, \Omega, \Omega^{-1}, \Omega a$ and $\rho(\Omega a)^{2}$ respectively, where $(a, \Omega)$ and the Reynolds number Re are selected as

$$
a=a_{1}, \quad \Omega=\left|\Omega_{1}\right|>0, \quad R e=\Omega a^{2} / \nu .
$$

For simplicity, any dimensionless quantity $g^{\prime}$ associated with $g$ is henceforth denoted by $g$. The fluid motion, free from body forces and of unknown velocity $\boldsymbol{u}$ and pressure 


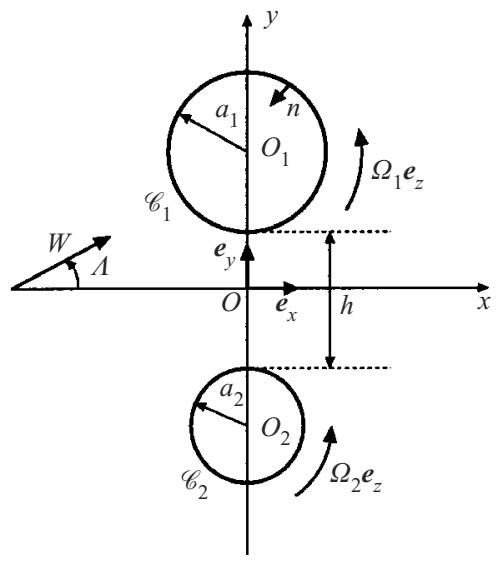

FIgURE 2. Two co-rotating circular cylinders $\left(\Omega_{1} \Omega_{2}>0\right)$ embedded in the external stream flow $\boldsymbol{W}=U \boldsymbol{e}_{x}+V \boldsymbol{e}_{y}$ of a viscous fluid.

$P$, is governed by the unsteady Navier-Stokes problem

$$
\begin{gathered}
\frac{\partial \boldsymbol{u}}{\partial t}+(\boldsymbol{u} \cdot \nabla) \boldsymbol{u}=-\nabla P+R e^{-1} \nabla^{2} \boldsymbol{u}, \nabla \cdot \boldsymbol{u}=0 \text { in } \mathscr{D}, \\
\boldsymbol{u}(\boldsymbol{x}, 0)=\mathbf{0} \text { in } \mathscr{D}, \\
H(t) \boldsymbol{W} \text { as }|\boldsymbol{x}| \rightarrow \infty, \quad \boldsymbol{u}(M)=H(t) \Omega_{n} \boldsymbol{e}_{z} \wedge \boldsymbol{O}_{n} \boldsymbol{M} \text { on } \mathscr{C}_{n},
\end{gathered}
$$

where $\mathscr{D}$ and $H$ respectively denote the steady multiply connected fluid domain and $H(t)$ the usual Heaviside step pseudo-function with $H(t)=1$ for $t>0$ and $H(t)=0$ otherwise.

The two-dimensional and divergence-free velocity field $\boldsymbol{u}$, of vorticity $\boldsymbol{w} \boldsymbol{e}_{z}$, may be deduced from the (modified) stream function $\psi(\boldsymbol{x}, t)$, determined up to an arbitrary function of $t$ by the relation $\boldsymbol{u}=\nabla \psi \wedge \boldsymbol{e}_{z}+\boldsymbol{W}$. Using Cartesian coordinates $(O, x, y)$ and setting

$$
\boldsymbol{u}=u \boldsymbol{e}_{x}+v \boldsymbol{e}_{y}, \quad \boldsymbol{W}=U \boldsymbol{e}_{x}+V \boldsymbol{e}_{y}, \quad W=|\boldsymbol{W}|=\left\{U^{2}+V^{2}\right\}^{1 / 2},
$$

the stream function $\psi$ is seen, from (2.2) and (2.4), to obey

$\left[\nabla^{2}-\operatorname{Re}\left(\frac{\partial}{\partial t}+U \frac{\partial}{\partial x}+V \frac{\partial}{\partial y}\right)\right] w=\operatorname{Re}[F]$ with $F:=\left[\frac{\partial \psi}{\partial y} \frac{\partial}{\partial x}-\frac{\partial \psi}{\partial x} \frac{\partial}{\partial y}\right] w$ in $\mathscr{D}$,

$$
u=U+\frac{\partial \psi}{\partial y}, \quad v=V-\frac{\partial \psi}{\partial x}, \quad w=-\nabla^{2} \psi \text { in } \mathscr{D},
$$

$\nabla \psi=\mathbf{0}$ and $w=0$ as $|\boldsymbol{x}| \rightarrow \infty, \quad \nabla \psi=H(t)\left[\boldsymbol{W} \wedge \boldsymbol{e}_{z}-\Omega_{n} \boldsymbol{O}_{n} \boldsymbol{M}\right]$ on $\mathscr{C}_{n}$.

The key term $F$ includes all nonlinear tems in $\psi$ entering equation (2.6), which is of unsteady Oseen form: as the Reynolds number $R e$ vanishes, inertial terms $R e[F]$ become negligible (recall the far-field behaviour (2.4)) in the outer domain $|\boldsymbol{x}|=O\left(R e^{-1}\right)$ where (2.6) immediately reduces to the unsteady Oseen equation. Upon introducing the modified vorticity $\zeta$ and function $f$ defined by

$$
\zeta(\boldsymbol{x}, t)=w(\boldsymbol{x}, t) \exp \{-\operatorname{Re}(U x+V y) / 2\}, \quad f(\boldsymbol{x}, t)=S(\boldsymbol{x}, t) \exp \{-\operatorname{Re}(U x+V y) / 2\}
$$


equations (2.6)-(2.7) yield, through elementary algebra, the relations

$$
\begin{gathered}
{\left[\nabla^{2}-\operatorname{Re}\left(\frac{\partial}{\partial t}+\operatorname{Re} \frac{W^{2}}{4}\right)\right] \zeta=\operatorname{Re}[f], \quad \nabla^{2} \psi=-\zeta \exp \{\operatorname{Re}(U x+V y) / 2\},} \\
f(\boldsymbol{x}, t)=\left[\frac{\partial \psi}{\partial y}\left(\operatorname{Re} \frac{U}{2}+\frac{\partial}{\partial x}\right)-\frac{\partial \psi}{\partial x}\left(\operatorname{Re} \frac{V}{2}+\frac{\partial}{\partial y}\right)\right] \zeta .
\end{gathered}
$$

Since the fluid domain is time-independent it is fruitful to use the Laplace transform. Defining the Laplace transform $\hat{g}$ of a function $g(x, t)$ as

$$
\hat{g}=\hat{g}(\boldsymbol{x} ; p)=\int_{0}^{\infty} \exp (-p t) g(\boldsymbol{x}, t) \mathrm{d} t \text { for } p>0,
$$

we obtain $\hat{H}(p)=1 / p$ and, since $(2.3)$ yields $\zeta(\boldsymbol{x}, 0)=w(\boldsymbol{x}, 0)=0$, we deduce that $p \hat{\zeta}$ is the Laplace transform of $\partial \zeta / \partial t$. Accordingly, taking the Laplace transform of (2.10) and (2.8) one readily arrives at the following one-parameter $(p)$ and steady problem:

$$
\begin{gathered}
{\left[\nabla^{2}-\operatorname{Re}\left(p+\operatorname{Re} \frac{W^{2}}{4}\right)\right] \hat{\zeta}=\operatorname{Re}[\hat{f}], \quad \nabla^{2} \hat{\psi}=-\hat{\zeta} \exp \{\operatorname{Re}(U x+V y) / 2\} \quad \text { in } \mathscr{D},} \\
\nabla \hat{\psi}=\nabla \hat{\zeta}=\mathbf{0} \text { and } \hat{\zeta}=0 \text { as }|\boldsymbol{x}| \rightarrow \infty, \quad \nabla \hat{\psi}=\frac{1}{p}\left[\boldsymbol{W} \wedge \boldsymbol{e}_{z}-\Omega_{n} \boldsymbol{O}_{n} \boldsymbol{M}\right] \text { on } \mathscr{C}_{n}
\end{gathered}
$$

System (2.13)-(2.14) naturally must be supplemented with the detailed and unfortunately rather cumbersome dependence of the coupling function $\hat{f}$ on $\hat{\psi}, \hat{\zeta}, R e$ and $\boldsymbol{W}$. For later purposes, let us replace each local equation (2.13) by its integral formulation counterpart. Noting that $\hat{\psi}$ is uniquely determined up to a constant, we can select $\hat{\psi}$ such that, by virtue of (2.13)-(2.14), it obeys a Poisson equation and vanishes with its gradient at infinity. Applying Green's theorem, one accordingly obtains at any point $\boldsymbol{x}$ in $\mathscr{D}$ the well-known integral representation

$$
\begin{aligned}
\hat{\psi}(\boldsymbol{x} ; p) & =-\frac{1}{2 \pi} \int_{\mathscr{D}} \hat{\zeta}(\boldsymbol{y} ; p) \exp \{\operatorname{Re}(\boldsymbol{W} \cdot \boldsymbol{y}) / 2\} \log |\boldsymbol{x}-\boldsymbol{y}| \mathrm{d} S(\boldsymbol{y}) \\
& -\frac{1}{2 \pi} \int_{\mathscr{C}}\left\{[\nabla \hat{\psi} \cdot \boldsymbol{n}](\boldsymbol{y} ; p) \log |\boldsymbol{x}-\boldsymbol{y}|-\hat{\psi}(\boldsymbol{y} ; p) \nabla_{y}[\log |\boldsymbol{x}-\boldsymbol{y}|] \cdot \boldsymbol{n}(\boldsymbol{y})\right\} \mathrm{d} l(\boldsymbol{y})
\end{aligned}
$$

where $\mathrm{d} l$ and $\boldsymbol{n}$ respectively denote the differential arclength and the unit normal on $\mathscr{C}=\mathscr{C}_{1} \cup \mathscr{C}_{2}$, which is outward with respect to the fluid domain (see figure 2). Furthermore, as established in Nakanishi et al. (1997), the Green function $G(\boldsymbol{x}, \boldsymbol{y})$ such that

$$
\nabla^{2} G-\operatorname{Re}\left(p+\operatorname{Re} \frac{W^{2}}{4}\right) G=-2 \pi \delta(\boldsymbol{x}-\boldsymbol{y}),
$$

with $\delta$ the usual two-dimensional Dirac pseudo-function, is

$$
G(\boldsymbol{x}, \boldsymbol{y})=K_{0}(c|\boldsymbol{x}-\boldsymbol{y}|), \quad c=\left\{\operatorname{Re}\left(p+\operatorname{Re} \frac{W^{2}}{4}\right)\right\}^{1 / 2}
$$

where $K_{0}$ denotes usual zeroth-order modified Bessel function of the second kind, as defined in Abramowitz \& Stegun (1968). Resorting again to Green's theorem, we thus 
obtain, again at any point $\boldsymbol{x}$ in $\mathscr{D}$, our second integral representation

$$
\begin{aligned}
\hat{\zeta}(\boldsymbol{x} ; p) & =-\frac{R e}{2 \pi} \int_{\mathscr{D}} \hat{f}(\boldsymbol{y} ; p) G(\boldsymbol{x}, \boldsymbol{y}) \mathrm{d} S(\boldsymbol{y}) \\
& +\frac{1}{2 \pi} \int_{\mathscr{C}}\left\{[\nabla \hat{\zeta} \cdot \boldsymbol{n}](\boldsymbol{y} ; p) G(\boldsymbol{x}, \boldsymbol{y})-\hat{\zeta}(\boldsymbol{y} ; p) \nabla_{y}[G(\boldsymbol{x}, \boldsymbol{y})] \cdot \boldsymbol{n}(\boldsymbol{y})\right\} \mathrm{d} l(\boldsymbol{y}) .
\end{aligned}
$$

The analysis advocated in this paper rests on the previous coupled integral formulations (2.15) and (2.18). The coupling function $\hat{f}$, which includes all the nonlinear terms, appears only on the right-hand side of (2.18) but it is in general of complicated form.

\section{Long-time behaviour of the viscous flow}

This key section establishes the governing boundary-value problem for the stream function $\hat{\psi}(\boldsymbol{x} ; p)$ in the inner domain $|\boldsymbol{x}|=O(1)$ as both the Reynolds number $R e$ and $p$ vanish. This step makes use of matched asymptotic expansions and the inner and outer approximations required are calculated in $\S 3.3$ which may be skipped in a first reading of the paper.

\subsection{Assumptions and notation}

Even for our simple geometry consisting of two circular cylinders it is not possible to analytically solve (2.13)-(2.14) or (2.14)-(2.15) and (2.18). However, for a vanishing Reynolds number it remains both possible and of prime interest to establish asymptotic estimates of $\hat{\psi}, \hat{\zeta}$ and of the hydrodynamic forces on the cylinders in terms of the small parameter $R e$. As mentioned in the introduction, this task has been carried out for steady (Umemura 1982; Lee \& Leal 1986) or unsteady (Nakanishi et al. 1997) flows about one or several non-rotating cylinder(s) for a Reynolds number scaled on the external velocity $W=|\boldsymbol{W}|$. In the present work, we assume that

$$
\epsilon=\operatorname{Re} / 2 \ll 1, \quad h \leqslant O(1),
$$

i.e. we confine ourselves to the case of sufficiently close cylinders. Because $\epsilon \rightarrow 0$, the required approximation of $\hat{\zeta}(\boldsymbol{x} ; p)$ is dictated, by virtue of (2.18), by the asymptotic behaviour of $K_{0}(c|\boldsymbol{x}-\boldsymbol{y}|)$ with

$$
c=c(p, \epsilon)=\left\{\epsilon\left(2 p+\epsilon W^{2}\right)\right\}^{1 / 2} .
$$

As explained in Nakanishi et al. (1997) and Ueda et al. (2001), one thus needs to distinguish five significant local domains, in the sense of Kida (1991) and Kida \& Take (1995), in the $(r, p)$-plane, where $r=|\boldsymbol{x}|$. These domains, depicted in figure 3 and labelled (I) to (V), are such that:

(i) $p=O\left(\epsilon^{-1}\right)$ in region (I) which, since $\hat{g}(\boldsymbol{x} ; p) \sim g(\boldsymbol{x}, 0)$ as $p \rightarrow \infty$, describes the early stages of the flow. Within this domain (2.13) reduces to the Laplace transform of the unsteady Stokes equation and becomes $\left[\nabla^{2}-2 \epsilon p\right] \hat{\zeta} \sim 0$.

(ii) $p$ is of order of unity in domains (II) and (III) where $r=O(1)$ and $r=O\left(\epsilon^{-1 / 2}\right)$ respectively. In addition, we obtain $\nabla^{2} \hat{\zeta} \sim 0$, i.e. a steady Stokes flow approximation in (II) and $\left[\tilde{\nabla}^{2}-2 p\right] \hat{\zeta} \sim 2 \hat{f}$ in (III) with $\tilde{\nabla}^{2}=\epsilon^{-1} \nabla^{2}$.

(iii) Finally, $p=O(\epsilon)$ in the inner domain (IV) where $r=O(1)$ and the outer domain (V) where $\boldsymbol{X}=\epsilon \boldsymbol{x}$ and $R=|\boldsymbol{X}|=O(1)$. It is also found from (2.13) that $\nabla^{2} \hat{\zeta} \sim 0$ in (IV) while $\left[\nabla_{o}^{2}-\left(2 p / \epsilon+W^{2}\right)\right] \hat{\zeta} \sim 2 \hat{f} / \epsilon$ with $\nabla_{o}^{2}=\epsilon^{-2} \nabla^{2}$ in region (V). In addition, region (IV)-(V) describes the required long-time behaviour of the flow 


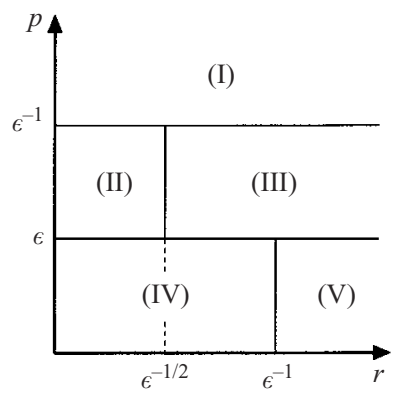

Figure 3. Distinguished significant local domains (I)-(V) in the $(r, p)$-plane.

because, for $\alpha>0$, one obtains (Sellier 1994)

$$
\hat{g}(\boldsymbol{x} ; p)=\frac{g^{\infty}(\boldsymbol{x})}{p}\left[1+O\left(p^{\alpha}\right)\right] \text { as } p \rightarrow 0 \text { if } g(\boldsymbol{x}, t)=g^{\infty}(\boldsymbol{x})\left[1+O\left(t^{-\alpha}\right)\right] \text { for } t \rightarrow \infty \text {. }
$$

3.2. Governing integral representations and assumptions for the long-time behaviour It should be possible, as achieved in Nakanishi et al. (1997) for one circular cylinder, to derive asymptotic estimates of $(\hat{\psi}, \hat{\zeta})$ in domains (I)- $(\mathrm{V})$ as $\epsilon$ vanishes. However, in this work we restrict the analysis to the long-time behaviour of the flow and thus consider $(\hat{\psi}, \hat{\zeta})$ in domains (IV) and (V) only. Within this framework, any function $g(\boldsymbol{x})$ is described in the inner (or outer) domain (IV) (or (V)) by its inner (or outer) representation $g_{i}(\boldsymbol{x})$ ) (or $g_{o}(\boldsymbol{X})$ ) and we denote by $\bar{g}_{i}$ (or $\bar{g}_{o}$ ) the typical magnitude of $g_{i}\left(\right.$ or $g_{o}$ ) and its derivatives with respect to $\boldsymbol{x}$ or $\boldsymbol{X}=\epsilon \boldsymbol{x}$ respectively. For instance, as $t \rightarrow \infty$ the unknown functions $\psi(\boldsymbol{x})$ and $\zeta(\boldsymbol{x})$ tend to $\psi^{\infty}(\boldsymbol{x})$ and $\zeta^{\infty}(\boldsymbol{x})$ respectively and inspection of $(2.11)$ readily yields

$$
\bar{f}_{i}^{\infty}=\bar{\psi}_{i}^{\infty} \bar{\zeta}_{i}^{\infty}, \quad \bar{f}_{o}^{\infty}=\epsilon^{2} \bar{\psi}_{o}^{\infty} \bar{\zeta}_{o}^{\infty} .
$$

Since $p=O(\epsilon) \ll 1$ the behaviour (3.3) suggests introducing quantities $\tilde{p}$, $\tilde{c}$ and functions $\psi^{\prime}, \zeta^{\prime}$ and $f^{\prime}$ such that

$$
\begin{aligned}
\tilde{p} & =p / \epsilon=O(1), \quad \tilde{c}=c / \epsilon=\left\{2 \tilde{p}+W^{2}\right\}^{1 / 2}=O(1), \\
\psi^{\prime}(\boldsymbol{x} ; \tilde{p}) & =p \hat{\psi}(\boldsymbol{x} ; p), \quad \zeta^{\prime}(\boldsymbol{x} ; \tilde{p})=p \hat{\zeta}(\boldsymbol{x} ; p), \quad f^{\prime}(\boldsymbol{x} ; \tilde{p})=p \hat{f}(\boldsymbol{x} ; p) .
\end{aligned}
$$

From (3.3)-(3.4) note that $\bar{\psi}_{i}^{\prime} \sim \bar{\psi}_{i}^{\infty}$ and $\bar{\zeta}_{i}^{\prime} \sim \bar{\zeta}_{i}^{\infty}$ in the inner domain (IV) whereas $\bar{\psi}_{o}^{\prime} \sim \bar{\psi}_{o}^{\infty}$ and $\bar{\zeta}_{o}^{\prime} \sim \bar{\zeta}_{o}^{\infty}$ in the outer domain (V) with

$$
\bar{f}_{i}^{\prime} \sim \bar{\psi}_{i}^{\prime} \bar{\zeta}_{i}^{\prime}, \quad \bar{f}_{o}^{\prime} \sim \epsilon^{2} \bar{\psi}_{o}^{\prime} \bar{\zeta}_{o}^{\prime}
$$

Clearly, any physical quantity of interest, such as the pointwise or global hydrodynamic force distributions experienced by the cylinders, or the flow topology in the vicinity of the two-cylinder cluster, will be deduced from the knowledge of $\psi_{i}^{\prime}(\boldsymbol{x} ; \tilde{p})$ for $|\boldsymbol{x}|=O(1)$. From the boundary condition (2.14) on $\mathscr{C}$ one obtains $\bar{\psi}_{i}^{\prime}=O(1)$. Thus, (2.13)-(2.14) in conjunction with (3.7) show that the unknown inner approximation $\psi_{i}^{\prime}$ obeys, for $|\boldsymbol{x}|=O(1)$ and up to order $\epsilon$,

$$
\nabla^{2} \zeta_{i}^{\prime}=0 \text { and } \nabla^{2} \psi_{i}^{\prime}=-\zeta_{i}^{\prime} \text { for }|\boldsymbol{x}|=O(1), \nabla \psi_{i}^{\prime}=\boldsymbol{W} \wedge \boldsymbol{e}_{z}-\Omega_{n} \boldsymbol{O}_{n} \boldsymbol{M} \text { on } \mathscr{C}_{n} .
$$

Since $h=O(1)$, the inner Stokes region indeed encompasses both circular cylinders. Note that it is not necessarily the case that $\bar{\zeta}_{i}^{\prime} \sim \bar{\psi}_{i}^{\prime}$ as perhaps suggested by (3.8): we only can say that $\bar{\zeta}_{i}^{\prime} \leqslant \bar{\psi}_{i}^{\prime}$ because $\psi_{i}^{\prime}$ may be split into potential and rotational 
contributions. At this stage, (3.8) is ill-posed: it must be supplemented with the proper behaviour of $\psi_{i}^{\prime}(\boldsymbol{x} ; \tilde{p})$ as $r=|\boldsymbol{x}| \rightarrow \infty$. This key far-field information is obtained by applying the method of matched asymptotic expansions (Van Dyke 1974) to the functions $\psi^{\prime}$ and $\zeta^{\prime}$. This procedure exploits the existence (Kaplun \& Lagerstrom 1957; Proudman \& Pearson 1957) of an overlap (intermediate) domain $r=O\left(\delta^{-1}\right)$, with $\epsilon \ll \delta \ll 1$, where both inner and outer approximations remain valid with $g(\boldsymbol{x} ; \tilde{p})=g_{i}(\overline{\boldsymbol{x}} / \delta ; \tilde{p})=g_{o}(\epsilon \overline{\boldsymbol{x}} / \delta ; \tilde{p})$ at any prescribed value of the intermediate variable $\overline{\boldsymbol{x}}=\delta \boldsymbol{x}$ for $|\overline{\boldsymbol{x}}|=O(1)$ and a fixed value of $\delta=\delta(\epsilon)=o(1)$. Omitting the dependence upon $\tilde{p}$, the functions $\psi^{\prime}, \zeta^{\prime}$ and $f^{\prime}$ are readily seen, from (3.6), (2.15) and (2.18), to obey the coupled integral representations

$$
\begin{aligned}
& \psi^{\prime}(\boldsymbol{x})\left.=-\frac{1}{2 \pi} \int_{\mathscr{D}} \zeta^{\prime}(\boldsymbol{y}) \exp \{\epsilon \boldsymbol{W} \cdot \boldsymbol{y})\right\} \log |\boldsymbol{x}-\boldsymbol{y}| \mathrm{d} S(\boldsymbol{y}) \\
&-\frac{1}{2 \pi} \int_{\mathscr{C}}\left\{\left[\nabla \psi^{\prime} \cdot \boldsymbol{n}\right](\boldsymbol{y}) \log |\boldsymbol{x}-\boldsymbol{y}|-\psi^{\prime}(\boldsymbol{y}) \nabla_{y}[\log |\boldsymbol{x}-\boldsymbol{y}|] \cdot \boldsymbol{n}(\boldsymbol{y})\right\} \mathrm{d} l(\boldsymbol{y}), \\
& \zeta^{\prime}(\boldsymbol{x})=-\frac{\epsilon}{\pi} \int_{\mathscr{D}} f^{\prime}(\boldsymbol{y}) K_{0}(\epsilon \tilde{c}|\boldsymbol{x}-\boldsymbol{y}|) \mathrm{d} S(\boldsymbol{y}) \\
&+\frac{1}{2 \pi} \int_{\mathscr{C}}\left\{\left[\nabla \zeta^{\prime} \cdot \boldsymbol{n}\right](\boldsymbol{y}) K_{0}(\epsilon \tilde{c}|\boldsymbol{x}-\boldsymbol{y}|)-\zeta^{\prime}(\boldsymbol{y}) \nabla_{y}\left[K_{0}(\epsilon \tilde{c}|\boldsymbol{x}-\boldsymbol{y}|)\right] \cdot \boldsymbol{n}(\boldsymbol{y})\right\} \mathrm{d} l(\boldsymbol{y}) .
\end{aligned}
$$

The next subsection derives both outer and inner approximations of $\left(\psi^{\prime}, \zeta^{\prime}\right)$ by appealing to (3.9)-(3.10) whereas $\S 3.4$ enforces the matching conditions and deduces the required far-field behaviour of $\psi_{i}^{\prime}$.

\subsection{Outer and inner approximations of $\left(\psi^{\prime}, \zeta^{\prime}\right)$}

We resort to polar coordinates $(r, \theta)$ and $(\rho, \varphi)$ for $\boldsymbol{x}$ and $\boldsymbol{y}$ respectively and denote by $I(g)$ and $L(g)$ the domain and boundary integrals arising on the right-hand sides of (3.9) or (3.10) for $g=\psi^{\prime}$ or $g=\zeta^{\prime}$.

Let us start with the outer approximations $\psi_{o}^{\prime}(\boldsymbol{X})$ and $\zeta_{o}^{\prime}(\boldsymbol{X})$ when $R=|\boldsymbol{X}|=$ $\epsilon r=O(1)$. Exploiting, for $r=|\boldsymbol{x}|>\rho=|\boldsymbol{y}|$, the basic expansion (deduced from Gradshteyn \& Ryzhik 1965, formula 1.514)

$$
\log |\boldsymbol{x}-\boldsymbol{y}|=\log r-\sum_{m \geqslant 1} \frac{1}{m}\left(\frac{\rho}{r}\right)^{m} \cos [m(\theta-\varphi)]
$$

we immediately obtain, for $\boldsymbol{X}=(R, \theta)$,

$$
L\left(\psi^{\prime}\right)=-\beta_{0}(\log R-\log \epsilon)-\sum_{m \geqslant 1} \epsilon^{m}\left[\beta_{m}^{c}(\epsilon) \cos m \theta+\beta_{m}^{s}(\epsilon) \sin m \theta\right] R^{-m}
$$

where the real quantities $\beta_{0}, \beta_{m}^{c}(\epsilon)$ and $\beta_{m}^{s}(\epsilon)$ are defined by

$$
\begin{gathered}
\beta_{0}=\frac{1}{2 \pi} \int_{\mathscr{C}} \frac{\partial \psi^{\prime}}{\partial n} \mathrm{~d} l(\boldsymbol{y})=\Omega_{1} a_{1}^{2}+\Omega_{2} a_{2}^{2}, \\
\beta_{m}^{c}+\mathrm{i} \beta_{m}^{s}=\frac{1}{2 \pi m} \int_{\mathscr{C}}\left\{\frac{\partial \psi^{\prime}}{\partial n} \rho^{m} \mathrm{e}^{\mathrm{i} m \varphi}-\psi^{\prime}(\boldsymbol{y}) \frac{\partial}{\partial n}\left[\rho^{m} \mathrm{e}^{\mathrm{i} m \varphi}\right]\right\} \mathrm{d} l(\boldsymbol{y}), m \geqslant 1 .
\end{gathered}
$$

Unlike the case of $\beta_{0}$, whose value is deduced from the boundary condition (3.8), the coefficients $\beta_{m}^{c}(\epsilon)$ and $\beta_{m}^{s}(\epsilon)$ are unknown (but of the order of $\bar{\psi}_{i}^{\prime}$ ). The quantity $L\left(\zeta^{\prime}\right)$ is expanded in a similar fashion by using, for $|\boldsymbol{y}|<|\boldsymbol{x}|$ and $b>0$, the summation 
theorem (Gradstheyn \& Ryzhik 1965, formula 8.531.2)

$$
K_{0}(b|\boldsymbol{x}-\boldsymbol{y}|)=K_{0}(b r) I_{0}(b \rho)+2 \sum_{m \geqslant 1} K_{m}(b r) I_{m}(b \rho) \cos [m(\theta-\varphi)],
$$

where $I_{0}, I_{m}$ and $K_{m}$ designate, for $m \geqslant 1$, the modified $m$ th-order Bessel functions of the first and second kind (Abramowitz \& Stegun 1965). The counterpart of (3.12) derived is

$$
L\left(\zeta^{\prime}\right)=\alpha_{0}(\epsilon) K_{0}(\tilde{c} R)+\sum_{m \geqslant 1} \epsilon^{m}\left[\alpha_{m}^{c}(\epsilon) \cos m \theta+\alpha_{m}^{s}(\epsilon) \sin m \theta\right] K_{m}(\tilde{c} R)
$$

with unknown and real coefficients $\alpha_{0}(\epsilon), \alpha_{m}^{c}(\epsilon)$ and $\alpha_{m}^{s}(\epsilon)$ such that

$$
\begin{gathered}
\alpha_{0}(\epsilon)=\frac{1}{2 \pi} \int_{\mathscr{C}}\left\{\frac{\partial \zeta^{\prime}}{\partial n} I_{0}(\epsilon \tilde{c} \rho)-\zeta^{\prime} \frac{\partial}{\partial n}\left[I_{0}(\epsilon \tilde{c} \rho)\right]\right\} \mathrm{d} l(\boldsymbol{y}) \\
\alpha_{m}^{c}(\epsilon)+\mathrm{i} \alpha_{m}^{s}(\epsilon)=\frac{\epsilon^{-m}}{\pi} \int_{\mathscr{C}}\left\{\frac{\partial \zeta^{\prime}}{\partial n} I_{m}(\epsilon \tilde{c} \rho) \mathrm{e}^{\mathrm{i} m \varphi}-\zeta^{\prime} \frac{\partial}{\partial n}\left[I_{m}(\epsilon \tilde{c} \rho) \mathrm{e}^{\mathrm{i} m \varphi}\right]\right\} \mathrm{d} l(\boldsymbol{y}), m \geqslant 1 .
\end{gathered}
$$

Because (Abramowitz \& Stegun 1965, p. 375)

$$
I_{0}(u)=1+\frac{u^{2}}{4}+O\left(u^{4}\right) \text { and } I_{m}(u)=\frac{u^{m}}{2^{m} m !}+O\left(u^{m+2}\right) \text { for } m \geqslant 1 \text { as } u \rightarrow 0,
$$

note that $\alpha_{0}(\epsilon), \alpha_{m}^{c}(\epsilon)$ and $\alpha_{m}^{s}(\epsilon)$ are of the order of $\bar{\zeta}_{i}^{\prime}$.

Unlike the previous cases of $L\left(\psi^{\prime}\right)$ and $L\left(\zeta^{\prime}\right)$, the treatment of the domain contributions $I\left(\psi^{\prime}\right)$ and $I\left(\zeta^{\prime}\right)$ turns out to be somewhat awkward. For conciseness, the expansion of $I\left(\zeta^{\prime}\right)$ is detailed in Appendix A, for sufficiently close cylinders $(h=O(1))$, and other domain integrals (for instance $I\left(\psi^{\prime}\right)$ but also the domain contributions to the inner approximations) are left to the reader, further details being given in Kida (1991), Kida \& Take (1995), Nakanishi et al. (1997) and Ueda et al. (2001). The exact (not approximated) outer expansions $\left(\psi_{o}^{\prime}, \zeta_{o}^{\prime}\right)$ obtained are

$$
\begin{aligned}
& \zeta_{o}^{\prime}(\boldsymbol{X})= \sum_{m \geqslant 1}\left\{\left[\epsilon^{m} \alpha_{m}^{c}(\epsilon)-A_{m}^{c}(\epsilon)\right] \cos m \theta+\left[\epsilon^{m} \alpha_{m}^{s}(\epsilon)-A_{m}^{s}(\epsilon)\right] \sin m \theta\right\} K_{m}(\tilde{c} R) \\
&+\left[\alpha_{0}(\epsilon)-A_{0}(\epsilon)\right] K_{0}(\tilde{c} R)-\frac{1}{\pi \epsilon} \mathrm{pf} \int_{\mathbb{R}^{2}} f_{o}^{\prime}(\boldsymbol{Y}) K_{0}(\tilde{c}|\boldsymbol{X}-\boldsymbol{Y}|) \mathrm{d} S(\boldsymbol{Y}), \\
& \psi_{o}^{\prime}(\boldsymbol{X})=-\sum_{m \geqslant 1}\left\{\left[\epsilon^{m} \beta_{m}^{c}(\epsilon)+C_{m}^{c}(\epsilon)\right] \cos m \theta+\left[\epsilon^{m} \beta_{m}^{s}(\epsilon)+C_{m}^{s}(\epsilon)\right] \sin m \theta\right\} R^{-m} \\
&-C_{0}^{\prime}(\epsilon)-\left[\beta_{0}+C_{0}(\epsilon)\right] \log R-\frac{1}{2 \pi \epsilon^{2}} \mathrm{pf} \int_{\mathbb{R}^{2}} \zeta_{o}^{\prime}(\boldsymbol{Y}) \exp (\boldsymbol{W} \cdot \boldsymbol{Y}) \log |\boldsymbol{X}-\boldsymbol{Y}| \mathrm{d} S(\boldsymbol{Y})
\end{aligned}
$$

where the symbol pf denotes the strongly singular integration in the finite part sense of Hadamard (Hadamard 1932; Sellier 1994, 1997) and the assumed but reasonable generalized expansions of the outer functions $f_{o}^{\prime}(\boldsymbol{Y})$ and $g_{o}^{\prime}(\boldsymbol{Y})=\zeta_{o}^{\prime}(\boldsymbol{Y}) \exp (\boldsymbol{W} \cdot \boldsymbol{Y})$ for $O(\epsilon / \delta) \leqslant|\boldsymbol{Y}| \ll 1$ (see (A 6) and (A 12)) adequately define those functions in the vicinity of the origin. Inspection of (3.20)-(3.21) suggests the following remarks:

(i) The expansion (3.21) readily shows that the inner behaviour of $\psi_{o}^{\prime}$ is free from any term proportional to $R^{2}$. From the matching principle, we thus prove that there 
is no solid-body rotation far from the cluster. This property has been suggested and prescribed by Watson $(1995,1996)$ on physical grounds.

(ii) As obtained by Nakanishi et al. (1997) for a simple circular cylinder, we expect (by virtue of (2.13)) that $\bar{\zeta}_{o}^{\prime} \sim \epsilon^{2} \bar{\psi}_{o}^{\prime}$ and $\bar{\zeta}_{o}^{\prime} \ll \epsilon$ (the analytical solution obtained in $\S 4$ will clearly satisfy these conditions). Since $\bar{f}_{o}^{\prime} / \epsilon \sim \epsilon \bar{\psi}_{o}^{\prime} \bar{\zeta}_{o}^{\prime}$, the integrals in (3.20) and (3.21) thus become negligible and significant respectively.

(iii) Any non-integral term arising on the right-hand side of (3.20) or (3.21) is seen to obey $\nabla_{o}^{2} F=0$ or $\nabla_{o}^{2} F=\tilde{c}^{2} F$ respectively if one sets $\nabla_{o}^{2}=\partial^{2} / \partial X^{2}+\partial^{2} / \partial Y^{2}$. Accordingly, the outer aproximations $\psi_{o}^{\prime}$ and $\zeta_{o}^{\prime}$ retained satisfy

$$
\left[\nabla_{o}^{2}-\tilde{c}^{2}\right] \zeta_{o}^{\prime}=0, \quad \epsilon^{2} \nabla_{o}^{2} \psi_{o}^{\prime}=-\zeta_{o}^{\prime} \exp (\boldsymbol{W} \cdot \boldsymbol{X}), \quad\left|\nabla \psi_{o}^{\prime}\right|=\left|\nabla \zeta_{o}^{\prime}\right|=\zeta_{o}^{\prime}=0 \text { as }|\boldsymbol{X}| \rightarrow \infty .
$$

Let us now construct inner approximations of $\psi^{\prime}$ and $\zeta^{\prime}$. Appealing to (3.11) our procedure yields, for $\boldsymbol{x}=(r, \theta)$ and $r=O(1)$, the decomposition

$$
\begin{aligned}
\psi_{i}^{\prime}(\boldsymbol{x}) & =-\frac{1}{2 \pi} \int_{\mathscr{C}}\left\{\frac{\partial \psi^{\prime}}{\partial n}(\boldsymbol{y}) \log |\boldsymbol{x}-\boldsymbol{y}|-\psi^{\prime}(\boldsymbol{y}) \frac{\partial}{\partial n}[\log |\boldsymbol{x}-\boldsymbol{y}|]\right\} \mathrm{d} l(\boldsymbol{y})-D_{0}(\epsilon) \\
& -\sum_{m \geqslant 1}\left[D_{m}^{c}(\epsilon) \cos m \theta+D_{m}^{s}(\epsilon) \sin m \theta\right] r^{m}-\operatorname{pf} \int_{\mathscr{D}} \zeta_{i}^{\prime}(\boldsymbol{y}) \exp (\epsilon \boldsymbol{W} \cdot \boldsymbol{y}) \frac{\log |\boldsymbol{x}-\boldsymbol{y}|}{2 \pi} \mathrm{d} S(\boldsymbol{y}) .
\end{aligned}
$$

As $|\boldsymbol{y}| \rightarrow \infty$, the quantity $g_{i}(\boldsymbol{y})=\zeta_{i}^{\prime}(\boldsymbol{y}) \exp (\epsilon \boldsymbol{W} \cdot \boldsymbol{y})$ here is defined by its generalized expansion for $|\boldsymbol{y}|=O\left(\delta^{-1}\right)$ and it may become strongly singular (the singularities occurring are taken into account, as indicated by the pf symbol). Of course, (3.23) fully agrees with the link $\nabla^{2} \psi_{i}^{\prime}=-\zeta_{i}^{\prime}$ in the inner domain. As $u \rightarrow 0$ one obtains $K_{0}(u)=-[\gamma+\log (u / 2)]+O\left(u^{2} \log u\right)$ with $\gamma$ the Euler constant. Using (3.9)-(3.10), (3.15) and (3.17), one thus obtains in the inner domain $|\boldsymbol{x}|=O$ (1)

$$
\begin{aligned}
L\left(\zeta^{\prime}\right)=- & \frac{1}{2 \pi} \int_{\mathscr{C}}\left\{\frac{\partial \zeta^{\prime}}{\partial n}(\boldsymbol{y}) \log |\boldsymbol{x}-\boldsymbol{y}|-\zeta^{\prime}(\boldsymbol{y}) \frac{\partial}{\partial n}[\log |\boldsymbol{x}-\boldsymbol{y}|]\right\} \mathrm{d} l(\boldsymbol{y}) \\
& -\left[\gamma+\log \left(\frac{1}{2} \epsilon \tilde{\boldsymbol{c}}\right)\right] \alpha_{0}(0)+O\left(\epsilon^{2} \log \epsilon \bar{\zeta}_{i}^{\prime}\right), \\
I\left(\zeta^{\prime}\right)= & \frac{\epsilon}{\pi} \mathrm{pf} \int_{\mathscr{D}} f_{i}^{\prime}(\boldsymbol{y})\left[\gamma+\log \left(\frac{1}{2} \epsilon \tilde{\boldsymbol{c}}\right)+\log |\boldsymbol{x}-\boldsymbol{y}|\right] \mathrm{d} S(\boldsymbol{y})-E_{0}^{\prime}(\epsilon) \\
& -\sum_{m \geqslant 1}\left[E_{m}^{\prime c}(\epsilon) \cos m \theta+E_{m}^{\prime s}(\epsilon) \sin m \theta\right] I_{m}(\epsilon \tilde{c} r)+O\left(\epsilon^{3} \log \epsilon{\overline{f_{i}}}_{i}^{\prime}\right) .
\end{aligned}
$$

Henceforth, we are content with approximating $\zeta_{i}^{\prime}=L\left(\zeta^{\prime}\right)+I\left(\zeta^{\prime}\right)$ up to the order of $\bar{\zeta}_{i}^{\prime}$. By virtue of (3.19) and because $\epsilon \bar{f}_{i}^{\prime} \sim \epsilon \bar{\zeta}_{i}^{\prime}$, this leading approximation, further denoted by $\zeta_{i}^{\prime}$, is found, from (3.24)-(3.25), to be

$$
\begin{aligned}
\zeta_{i}^{\prime}(\boldsymbol{x}) & =-\frac{1}{2 \pi} \int_{\mathscr{C}}\left\{\frac{\partial \zeta^{\prime}}{\partial n}(\boldsymbol{y}) \log |\boldsymbol{x}-\boldsymbol{y}|-\zeta^{\prime}(\boldsymbol{y}) \frac{\partial}{\partial n}[\log |\boldsymbol{x}-\boldsymbol{y}|]\right\} \mathrm{d} l(\boldsymbol{y}) \\
& -\left[\gamma+\log \left(\frac{1}{2} \epsilon \tilde{c}\right)\right] \alpha_{0}(0)-E_{0}(\epsilon)-\sum_{m \geqslant 1}\left[E_{m}^{c}(\epsilon) \cos m \theta+E_{m}^{s}(\epsilon) \sin m \theta\right] r^{m}
\end{aligned}
$$

with retained coefficients $E_{0}, E_{m}^{c, s}$ of order of $\bar{\zeta}_{i}^{\prime}$.

In summary, the approximations of $\left(\psi_{i}^{\prime}, \zeta_{i}^{\prime}\right)$ and $\left(\psi_{o}^{\prime}, \zeta_{o}^{\prime}\right)$, in the inner and outer domain respectively, are given by (3.23), (3.26), (3.21) and (3.20) where, under our previous assumption (ii), $f_{o}^{\prime}$ is set to zero. The governing and unknown coefficients 
$\alpha_{0}(0), \alpha_{m}^{c, s}(\epsilon), A_{0}(\epsilon)$ and $A_{m}^{c, s}(\epsilon), D_{0}(\epsilon), D_{m}^{c, s}(\epsilon), E_{0}(\epsilon), E_{m}^{c, s}(\epsilon), C_{0}^{\prime}(\epsilon), C_{0}(\epsilon), C_{m}^{c, s}(\epsilon)$, $\beta_{m}^{c, s}(\epsilon)$ will be obtained, up to the order of approximation addressed, by enforcing the matching rules (Van Dyke 1974) and the boundary condition (3.8) on each cylinder $\mathscr{C}_{n}$. This matching process is achieved, both for $\psi^{\prime}$ and $\zeta^{\prime}$, in the next subsection $\S 3.4$.

\subsection{Matching and inner problem for the stream function}

In this subsection we derive the leading approximations of $\zeta_{i}^{\prime}$ and $\zeta_{o}^{\prime}$ and exploit the results obtained to establish the required far-field behaviour of the Stokes approximation $\psi_{i}^{\prime}$. For clarity, the procedure is briefly explained and most of the calculations are displayed in Appendix B.

Recalling that our approximation $\zeta_{i}^{\prime}$ extends to the intermediate domain $|\boldsymbol{x}|=$ $O\left(\delta^{-1}\right) \rightarrow \infty$, one deduces from (3.26) and by matching the inner and outer expansions of $\zeta^{\prime}$ (see Appendix B) that

$$
\zeta_{i}^{\prime}(\boldsymbol{x})=-\alpha_{0}(0)[\log r+1]-[\gamma-1+\log (\epsilon \tilde{c} / 2)] \alpha_{0}(0)-E_{0}(\epsilon)+o\left(\bar{\zeta}_{i}^{\prime}\right) \text { as } r \rightarrow \infty .
$$

The first term on the right-hand side of (3.27) induces the contribution $\alpha_{0}(0) r^{2} \log r / 4$ in the far-field behaviour of $\psi_{i}^{\prime}$ (this latter function fulfils $\nabla^{2} \psi_{i}^{\prime}=-\zeta_{i}^{\prime}$ in the overlap domain and no harmonic function behaves as $G=r^{2} \log r$ as $r$ increases). As the pressure associated with $G$, within the Stokes approximation, is not single-valued we thus necessarily have $\alpha_{0}(0)=0$. Finally, one arrives at the key approximations (see Appendix B)

$$
\begin{gathered}
\zeta_{i}^{\prime}(\boldsymbol{x})=-\frac{1}{2 \pi} \int_{\mathscr{C}}\left\{\frac{\partial \zeta^{\prime}}{\partial n}(\boldsymbol{y}) \log |\boldsymbol{x}-\boldsymbol{y}|-\zeta^{\prime}(\boldsymbol{y}) \frac{\partial}{\partial n}[\log |\boldsymbol{x}-\boldsymbol{y}|]\right\} \mathrm{d} l(\boldsymbol{y}) \text { with } \alpha_{0}(0)=0, \\
\zeta_{o}^{\prime}(\boldsymbol{X})=\epsilon\left[\alpha_{1}^{c}(0) \cos \theta+\alpha_{1}^{s}(0) \sin \theta\right] K_{1}(\tilde{c} R) .
\end{gathered}
$$

Substituting (3.29) into (3.21) it is thus possible to match the expansions (3.21)-(3.23) and to deduce the required far-field behaviour of $\psi_{i}^{\prime}$, as detailed in Appendix B. In summary, one finally obtains the well-posed inner problem

$$
\begin{gathered}
\nabla^{4} \psi_{i}^{\prime}=0, \quad \nabla \psi_{i}^{\prime}=\boldsymbol{W} \wedge \boldsymbol{e}_{z}-\Omega_{n} \boldsymbol{O}_{n} \boldsymbol{M} \text { on } \mathscr{C}_{n}, \\
\psi_{i}^{\prime}=-P x \log r+Q y \log r+E \log r+A x+B y+O(1) \text { as }|\boldsymbol{x}| \rightarrow \infty
\end{gathered}
$$

with the following relations:

$$
\begin{gathered}
P=\frac{\alpha_{1}^{c}(0)}{2 \tilde{c}}, \quad Q=-\frac{\alpha_{1}^{s}(0)}{2 \tilde{c}}, \quad E=-\operatorname{pf} \int_{\mathscr{D}} \frac{\zeta_{i}^{\prime}(\boldsymbol{y}) \exp (\epsilon \boldsymbol{W} \cdot \boldsymbol{y}) \mathrm{d} S(\boldsymbol{y})}{2 \pi}-\beta_{0}, \\
A=\operatorname{pf} \int_{\mathbb{R}^{2}} \frac{\zeta_{o}^{\prime}(\boldsymbol{Y}) \exp (\boldsymbol{W} \cdot \boldsymbol{Y}) \cos \varphi}{2 \pi \epsilon|\boldsymbol{Y}|} \mathrm{d} S(\boldsymbol{Y})+\frac{\alpha_{1}^{c}(0)}{2 \tilde{c}}\left[\frac{1}{2}-\log \epsilon\right], \\
B=\operatorname{pf} \int_{\mathbb{R}^{2}} \frac{\zeta_{o}^{\prime}(\boldsymbol{Y}) \exp (\boldsymbol{W} \cdot \boldsymbol{Y}) \sin \varphi}{2 \pi \epsilon|\boldsymbol{Y}|} \mathrm{d} S(\boldsymbol{Y})+\frac{\alpha_{1}^{s}(0)}{2 \tilde{c}}\left[\frac{1}{2}-\log \epsilon\right] .
\end{gathered}
$$

Note that system (3.30)-(3.31) is consistent with $\alpha_{0}(0)=0$. The Stokeslet $(Q y-$ $P x) \log r$, the only term that induces a logarithmic divergence of the velocity at infinity, is closely related to the total force per unit length experienced by the twocylinder cluster. More precisely, exploiting the inner Stokes flow approximation, one may easily check that the Laplace transform, $\hat{R}_{x}(p) \boldsymbol{e}_{x}+\hat{R}_{y}(p) \boldsymbol{e}_{y}$, of this dimensional 
total force is such that

$$
p \hat{R}_{x}(p)=4 \pi \mu \Omega a Q=-\frac{2 \pi \mu \Omega a}{\tilde{c}} \alpha_{1}^{s}(0), \quad p \hat{R}_{y}(p)=4 \pi \mu \Omega a P=\frac{2 \pi \mu \Omega a}{\tilde{c}} \alpha_{1}^{c}(0) .
$$

The constant $E$ occurring in (3.31) is related to the Laplace transforms $\hat{\Gamma}(p)$ and $\hat{M}(p)$ of the dimensional circulation and torque (about $O$ ) per unit length about the cylinders as follows:

$$
p \hat{\Gamma}(p)=-2 \pi \Omega a^{2} E, \quad p \hat{M}(p)=4 \pi \mu \Omega a^{2} E .
$$

Finally, the sum $A x+B y$ induces the uniform velocity $B \boldsymbol{e}_{x}-A \boldsymbol{e}_{y}$ far from the cylinders.

Introducing the angle $\Lambda$, as in figure 2 , by $\boldsymbol{W} / W=\cos \Lambda \boldsymbol{e}_{x}+\sin \Lambda \boldsymbol{e}_{y}$ and substituting (3.29) into (3.33)-(3.34) yields, as detailed in Appendix C,

$$
\begin{aligned}
& A=\frac{\alpha_{1}^{c}(0)}{2 \tilde{c}}\left[k_{0}+k_{2} \cos 2 \Lambda-\log \epsilon+\frac{1}{2}\right]+\frac{\alpha_{1}^{s}(0)}{2 \tilde{c}} k_{2} \sin 2 \Lambda, \\
& B=\frac{\alpha_{1}^{c}(0)}{2 \tilde{c}} k_{2} \sin 2 \Lambda+\frac{\alpha_{1}^{s}(0)}{2 \tilde{c}}\left[k_{0}-k_{2} \cos 2 \Lambda-\log \epsilon+\frac{1}{2}\right],
\end{aligned}
$$

where $k_{0}$ and $k_{2}$, which depend only upon $\boldsymbol{W}=(W, \Lambda)$ and $\tilde{c}$, are defined by $(\mathrm{C} 6)$. Noting that our definition (3.32) of $E$ is automatically satisfied, from (3.13) and the relation $\nabla^{2} \psi^{\prime}=-\zeta^{\prime} \exp (\epsilon \boldsymbol{W} \cdot \boldsymbol{y})$ in the fluid domain, the key far-field behaviour (3.31) appears as a two-parameter asymptotics governed by the unknown coefficients $\alpha_{1}^{c}(0) / \tilde{c}$ and $\alpha_{1}^{s}(0) / \tilde{c}$. These quantities obey

$$
\frac{\alpha_{1}^{c}(0)+\mathrm{i} \alpha_{1}^{s}(0)}{(2 \pi)^{-1} \tilde{c}}=\int_{\mathscr{C}}\left\{\frac{\partial \zeta_{i}^{\prime}}{\partial n}(\boldsymbol{y}) \rho \mathrm{e}^{\mathrm{i} m \varphi}-\zeta_{i}^{\prime}(\boldsymbol{y}) \frac{\partial}{\partial n}\left(\rho \mathrm{e}^{\mathrm{i} m \varphi}\right)\right\} \mathrm{d} l(\boldsymbol{y}), \quad \text { with } \zeta_{i}^{\prime}=-\nabla^{2} \psi_{i}^{\prime}
$$

and they directly govern the outer vorticity approximation (3.29) and the total force and torque acting on the cluster (see (3.35)-(3.36)). Solving the well-posed inner problem (3.30)-(3.31), which constitutes the main result of $\S 3$, we will obtain both $\alpha_{1}^{c}(0) / \tilde{c}$ and $\alpha_{1}^{s}(0) / \tilde{c}$. Before carrying out this treatment for two circular cylinders, let us emphasize that all the material developed in $\S 2$ and $\S 3$ also readily extends to the case of any $N$-cylinder cluster of sufficiently close and either rotating circular cylinders or motionless but arbitrarily shaped bodies embedded in a uniform flow $\boldsymbol{W}$. Only such situations comply with a time-independent fluid domain.

\section{Solution for a two-cylinder cluster}

This section addresses in detail the case of two rotating circular cylinders, as mentioned in the introduction. Such a simple geometry not only permits us to derive the analytical solution of (3.30)-(3.31) but it also allows us to discuss Jeffery's paradox by letting $\boldsymbol{W}$ and $\tilde{p}$ vanish as appropriate.

\subsection{The analytical solution}

Following the pioneering papers of Jeffery $(1920,1922)$, we resort to bipolar coordinates $(\xi, \eta)$ related to Cartesian coordinates $(x, y)$ by

$$
x=\frac{d \sin \eta}{\cosh \xi-\cos \eta}, \quad y=\frac{d \sinh \xi}{\cosh \xi-\cos \eta}
$$

with $d>0,-\pi / 2<\eta \leqslant \pi / 2$ and $\xi$ either positive or negative. The fluid occupies the domain $-\beta<\xi<\alpha$ with circular boundaries $\mathscr{C}_{1}$ and $\mathscr{C}_{2}$ with equations $\xi=\alpha>0$ 
and $\xi=-\beta<0$ respectively under the relations

$$
\sinh \alpha=\frac{d}{a_{1}}, \quad \sinh \beta=\frac{d}{a_{2}}, \quad \operatorname{coth} \alpha+\operatorname{coth} \beta=\frac{h_{1}+h_{2}}{d}
$$

that uniquely determine $(d, \alpha, \beta)$ in terms of our data $\left(a_{1}, a_{2}, h_{1}+h_{2}\right)$. Note that we accordingly locate $O$ so that $h_{1}=d \operatorname{coth} \alpha$ (and, consequently, $h_{2}=d \operatorname{coth} \beta$ ). The general solution to (3.30) is immediately obtained by appealing to Watson (1995, 1996) whose notation is henceforth adopted for convenience. One thus obtains

$$
\psi_{i}^{\prime}(\xi, \eta)=V x-U y+\frac{d}{\cosh \xi-\cos \eta}\left[\phi^{(0)}+B_{1} \phi^{(1)}+B_{2} \phi^{(2)}+B_{3} \phi^{(3)}\right]
$$

where functions $\phi^{(0)}, \ldots, \phi^{(2)}$, introduced in Watson (1996), fulfil the boundary conditions

$$
\begin{aligned}
& \phi^{(k)}=\delta_{3 k}^{\prime} P_{k}[\cosh \alpha-\cos \eta], \quad \frac{\partial \phi^{(k)}}{\partial \xi}=\delta_{3 k}^{\prime}\left[P_{k} \sinh \alpha+\delta_{k 0} \frac{d \Omega_{1}}{\sinh \alpha}\right] \text { at } \xi=\alpha, \\
& \phi^{(k)}=\delta_{3 k}^{\prime} Q_{k}[\cosh \beta-\cos \eta], \quad \frac{\partial \phi^{(k)}}{\partial \xi}=-\delta_{3 k}^{\prime}\left[Q_{k} \sinh \beta+\delta_{k 0} \frac{d \Omega_{2}}{\sinh \beta}\right] \text { at } \xi=-\beta,
\end{aligned}
$$

for $\delta_{i j}^{\prime}=1-\delta_{i j}$ if $\delta_{i j}$ denotes the usual Kronecker Delta, $k \in\{0, \ldots, 2\}$ and undetermined constants $P_{0}, P_{1}, P_{2}$ and $Q_{0}, Q_{1}, Q_{2}$. If needed (for instance when looking at the pointwise fluid velocity distribution), the above relations provide (Watson 1996) five constants among $P_{0}, P_{1}, P_{2}, Q_{0}, Q_{1}, Q_{2}$ (the stream function $\psi_{i}^{\prime}$ is determined up to a constant). At this stage, we need to enforce the two-parameter behaviour (3.31) in order to evaluate the unknown coefficients $\alpha_{1}^{c}(0) / \tilde{c}, \alpha_{1}^{s}(0) / \tilde{c}, B_{1}, B_{2}$ and $B_{3}$. From Watson (1996), the far-field asymptotics of (4.3) is

$$
\begin{aligned}
\psi_{i}^{\prime} & =\left\{V+B_{3}\left[2 \log (2 d)+T^{*}\right]\right\} x+\left\{T_{0}+B_{1} T_{1}-U+B_{2}\left[2 \log (2 d)+T_{2}\right]\right\} y \\
& -2 B_{3} x \log r-2 B_{2} y \log r+\left[S_{0}+B_{1} S_{1}+B_{2} S_{2}\right] r^{2} /(2 d)+O(\log r) \text { as } r \rightarrow \infty,
\end{aligned}
$$

where any constant $S_{0}, S_{1}, S_{2}, T_{0}, T_{1}, T_{2}$ or $T^{*}$ that occurs is solely expressed in terms of $\left(d, \alpha, \beta, \Omega_{1}, \Omega_{2}\right)$ and available in Watson $(1995,1996)$. Comparing (4.6) and (3.31), using our relations (3.32) and (3.37)-(3.38), yields

$$
\left.\begin{array}{l}
B_{1}=-\frac{S_{0}}{S_{1}}-\frac{S_{2}}{S_{1}} \frac{\alpha_{1}^{s}(0)}{4 \tilde{c}}, \quad B_{2}=\frac{\alpha_{1}^{s}(0)}{4 \tilde{c}}, \quad B_{3}=\frac{\alpha_{1}^{c}(0)}{4 \tilde{c}}, \\
\frac{\alpha_{1}^{c}(0)}{2 \tilde{c}}=\frac{\left(T_{0}-U-S_{0} T_{1} / S_{1}\right) k_{2} \sin 2 \Lambda-V k_{-}}{k_{2}^{2} \sin ^{2} 2 \Lambda-k_{-} k_{+}}, \\
\frac{\alpha_{1}^{s}(0)}{2 \tilde{c}}=\frac{V k_{2} \sin 2 \Lambda-\left(T_{0}-U-S_{0} T_{1} / S_{1}\right) k_{+}}{k_{2}^{2} \sin ^{2} 2 \Lambda-k_{-} k_{+}}
\end{array}\right\}
$$

if one adopts the following definitions:

$$
\begin{gathered}
k_{-}=k_{0}-k_{2} \cos 2 \Lambda-\log \epsilon+\frac{1}{2}-\log (2 d)-\frac{T_{2}}{2}+\frac{S_{2}}{2 S_{1}} T_{1}, \\
k_{+}=k_{0}+k_{2} \cos 2 \Lambda-\log \epsilon+\frac{1}{2}-\log (2 d)-\frac{T^{*}}{2} .
\end{gathered}
$$


Because $k_{0}=O(1)$ and $k_{2}=O(1)$ we obtain, as previously stated, $\alpha_{1}^{c, s}(0) / \tilde{c}=$ $O(1 / \log \epsilon) \ll \epsilon$. In addition (Watson 1995, 1996), the Laplace transform $\hat{R}_{x}^{n}(p) \boldsymbol{e}_{x}+$ $\hat{R}_{y}^{n}(p) \boldsymbol{e}_{y}$ of the force per unit length on the cylinder $\mathscr{C}_{n}$, is given by

$$
\begin{gathered}
p \hat{R}_{x}^{n}(p)=4 \pi \mu \Omega a\left\{(-1)^{n}\left[d_{0}^{(0)}-\frac{S_{0}}{S_{1}} d_{0}^{(1)}\right]-\frac{\alpha_{1}^{s}(0)}{4 \tilde{c}}\left[1-(-1)^{n}\left(d_{0}^{(2)}-\frac{S_{2}}{S_{1}} d_{0}^{(1)}\right)\right]\right\}, \\
p \hat{R}_{y}^{n}(p)=4 \pi \mu \Omega a \frac{\alpha_{1}^{c}(0)}{4 \tilde{c}}\left[1-(-1)^{n} d_{1}^{(3)}\right],
\end{gathered}
$$

where constants $d_{0}^{(0)}, d_{0}^{(1)}, d_{0}^{(1)}$ and $d_{1}^{(3)}$ depend solely upon $\left(d, \alpha, \beta, \Omega_{1}, \Omega_{2}\right)$ and are

$$
\begin{aligned}
d_{0}^{(1)}=- & \frac{4 \sinh ^{2} \alpha \sinh ^{2} \beta}{\Delta}, \frac{\Delta}{\alpha+\beta}=\frac{2 \sinh (\alpha+\beta) \sinh \alpha \sinh \beta}{\alpha+\beta}+\sinh ^{2} \alpha+\sinh ^{2} \beta, \\
& d_{0}^{(0)}=d \sinh (\alpha+\beta)\left[\Omega_{1} \sinh ^{2} \beta+\Omega_{2} \sinh ^{2} \alpha\right] /\{\Delta \sinh \alpha \sinh \beta\}, \\
& d_{0}^{(2)}=\left\{4 \sinh (\alpha-\beta) \sinh \alpha \sinh \beta+(\beta-\alpha)\left(\sinh ^{2} \alpha+\sinh ^{2} \beta\right)\right\} / \Delta, \\
d_{1}^{(3)}= & \{(\beta-\alpha) \cosh (\alpha+\beta)-\sinh (\beta-\alpha)\} /\{(\alpha+\beta) \cosh (\alpha+\beta)-\sinh (\alpha+\beta)\} .
\end{aligned}
$$

The above coefficients are easily computed by introducing $\eta=(\sinh \alpha)^{2}$ and solving the equation (see (4.2))

$$
a_{1}(1+\eta)^{1 / 2}+a_{2}\left[1+\left(a_{1} / a_{2}\right)^{2} \eta\right]^{1 / 2}=a_{1}+a_{2}+h,
$$

where $h=h_{1}+h_{2}-\left(a_{1}+a_{2}\right)$ is the gap between the cylinders. The required solution is

$$
\eta=-1+\frac{1}{4}\left\{1+\frac{a_{2}}{a_{1}}+\frac{h}{a_{1}}+\left[1-\left(\frac{a_{2}}{a_{1}}\right)^{2}\right] /\left(1+\frac{a_{2}}{a_{1}}+\frac{h}{a_{1}}\right)\right\}^{2}
$$

and when implementing (4.13)-(4.16) one finally makes use of the relations

$$
d=a_{1} \sqrt{\eta}, \quad \alpha=\log \{\sqrt{\eta}+\sqrt{1+\eta}\}, \quad \beta=\log \left\{\frac{a_{1}}{a_{2}} \sqrt{\eta}+\left[1+\left(\frac{a_{1}}{a_{2}}\right)^{2} \eta\right]^{1 / 2}\right\} .
$$

Not surprisingly, (4.11)-(4.12) agree with (3.35). If $\alpha_{1}^{c}(0) \neq 0$, it follows from (4.12) that

$$
r_{n}=\frac{p \hat{R}_{y}^{n}(p)}{p \hat{R}_{y}^{1}(p)+p \hat{R}_{y}^{2}(p)}=\frac{1}{2}\left[1-(-1)^{n} d_{1}^{(3)}\right] .
$$

Thus, the ratio of $\hat{R}_{y}^{n}$ to the sum $\hat{R}_{y}^{1}+\hat{R}_{y}^{2}$ is insensitive both to $p$ and to the angular velocities $\Omega_{1}$ and $\Omega_{2}$; it depends solely upon the cluster geometry $\left(a_{1}, a_{2}, h\right)$.

The functions $r_{1}$ and $r_{2}$ are plotted in figure 4 versus the gap $h$ and for different parameter settings $a_{2} \leqslant a_{1}=1$ (for $a_{2}>a_{1}$, one only needs to switch subscripts 1 and 2). Both functions $r_{1}$ and $r_{2}$ asymptote to $1 / 2$ as $h$ increases (we keep $h=O(1)$ in figure 4) and $r_{1}$ and $r_{2}$ increases and decreases respectively as $a_{1} / a_{2}$ increases or $h$ decreases. 


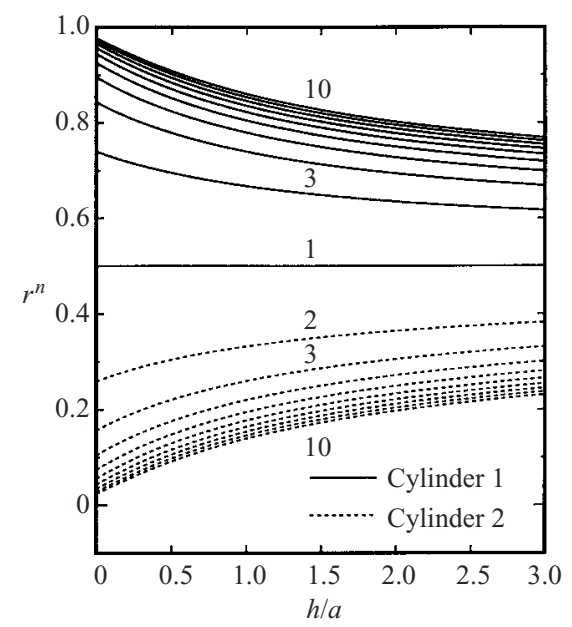

FIGURE 4. Coefficients $r_{1}$ (solid curves) and $r_{2}$ (dashed curves) for a few integer values of $a_{1} / a_{2} \geqslant 1$ and $a_{1}=1$. For $a_{2}=a_{1}, r_{1}=r_{2}$. Numbers next to curves indicate the ratio $a_{1} / a_{2}$.

\subsection{The steady limits and Jeffery's paradox}

This subsection examines the long-time flow induced by the rotation of two circular cylinders in the case of a zero outer stream flow, i.e. when both $\boldsymbol{W}=(W, \lambda)$ and $\tilde{p}$ vanish. As alluded to in the introduction, one may consider two different procedures in addressing this key issue: we first let $\tilde{p}$ go to zero and then make $W$ decrease (Case 1) or we first set $\boldsymbol{W}=\mathbf{0}$ and then let $\tilde{p}$ vanish (Case 2). As explained below, these cases actually yield different steady flows: $W$ must be non-zero in Case 1 whereas $\boldsymbol{W}=\mathbf{0}$ in Case 2.

\section{Case 1: $\tilde{p} \rightarrow 0$}

For this case we take $\tilde{p} \rightarrow 0$ and $W$ non-zero. In order to include the case of nonrotating cylinders we select as velocity scale $a \Omega^{\prime}$ with $\Omega^{\prime}=\operatorname{Max}\left(\left|\Omega_{1}\right|,\left|\Omega_{2}\right|, W / a\right)$. A straightforward analysis gives

$$
k_{ \pm}=-\frac{1}{2}\left[1_{-}^{+} \cos 2 \Lambda\right] \log \tilde{p}-\log \epsilon+O(1), \quad k_{2} \sim-\frac{1}{2}[1+\log 2+\log \tilde{p}]+\log W .
$$

Accordingly, the net force per unit length on $\mathscr{C}_{n}$ asymptotes to $\boldsymbol{R}^{n}=R_{x}^{n} \boldsymbol{e}_{x}+R_{y}^{n} \boldsymbol{e}_{y}$ as $t \rightarrow \infty$ with the leading-order estimates

$$
\begin{gathered}
\frac{R_{x}^{n}}{4 \pi \mu \Omega^{\prime} a} \sim-\frac{\cos \Lambda}{2 \log \epsilon}\left[1-(-1)^{n}\left(d_{0}^{(2)}-\frac{S_{2}}{S_{1}} d_{0}^{(1)}\right)\right]\left[W-\cos \Lambda\left(T_{0}-\frac{S_{0} T_{1}}{S_{1}}\right)\right], \\
\frac{R_{y}^{n}}{4 \pi \mu \Omega^{\prime} a} \sim-\frac{\sin \Lambda}{2 \log \epsilon}\left[1-(-1)^{n} d_{1}^{(3)}\right]\left[W-\cos \Lambda\left(T_{0}-\frac{S_{0} T_{1}}{S_{1}}\right)\right] .
\end{gathered}
$$

Note that (4.22)-(4.23) agree with Watson (1996) and extend to rotating cylinders the zeroth-order results of Lee \& Leal (1986) (if neither $\mathscr{C}_{1}$ nor $\mathscr{C}_{2}$ rotates then $\Omega^{\prime} a=W, T_{0}=S_{0}=0$ from Watson (1995) and one immediately recovers the results of Lee \& Leal (1986)). At the retained order of approximation, rotating but identical circular cylinders experience zero lift and equal drag forces expressed solely in terms of $(W, \Lambda)$ and $T_{0}=d\left(\Omega_{2}-\Omega_{1}\right) /[2 \sinh 2 \alpha]$ (if $a_{1}=a_{2}$ then $d_{0}^{(2)}=d_{1}^{(3)}=0$ and, see Watson (1995), $S_{2}=0$ ). In addition, for identical cylinders of equal angular velocities 
$\left(\Omega_{2}=\Omega_{1}\right)$ this non-zero drag on each cylinder is that prevailing for non-rotating cylinders $\left(T_{0}=0\right)$. In general, non-identical cylinders experience unequal and non-zero lift and drag forces but the net force $\boldsymbol{R}^{1}+\boldsymbol{R}^{2}$ on the two-cylinder cluster obeys

$$
W\left[\boldsymbol{R}^{1}+\boldsymbol{R}^{2}\right] \sim-\left[\frac{4 \pi \mu \Omega^{\prime} a}{\log \epsilon}\right]\left[W-\cos \Lambda\left(T_{0}-\frac{S_{0} T_{1}}{S_{1}}\right)\right] \boldsymbol{W} .
$$

Hence, the total leading-order lift force is zero and the total drag depends upon $(W, \Lambda)$ and the parameter setting $\left(a_{1}, a_{2}, h, \Omega_{1}, \Omega_{2}\right)$ through the coefficient $T_{0}-S_{0} T_{1} / S_{1}$ only. If $\boldsymbol{W}$ is normal $(\sin \Lambda=0)$ or parallel $(\cos \Lambda=0)$ to the line of centres the lift on each cylinder vanishes. In addition, the drag force on each cylinder does not depend upon the angular velocities $\Omega_{1}$ and $\Omega_{2}$ (as is the case for $d_{1}^{(3)}$ ) if $|\Lambda|=\pi / 2$ because (4.23) becomes

$$
R_{y}^{n} \sim-\frac{4 \pi \mu \Omega^{\prime} a W \Lambda}{|\Lambda| \log \epsilon} r_{n}
$$

with functions $r_{n}$ previously introduced in (4.20) and plotted in figure 4. Finally, if $\mathscr{C}_{1}$ and/or $\mathscr{C}_{2}$ rotate(s) the term $T_{0}-S_{0} T_{1} / S_{1}$ becomes non-zero and neither $R_{x}^{n}$ nor $R_{y}^{n}$ admit a limit as $\boldsymbol{W} \rightarrow \mathbf{0}$ (since this limit would depend upon $\Lambda$ ). Consequently, our Case 1 is not able to handle Jeffery's paradox since it fails in predicting a long-time flow that is quiescent far from the cylinders.

Case 2: $\boldsymbol{W} \rightarrow \mathbf{0}$

Now we assume first that $\boldsymbol{W} \rightarrow \mathbf{0}$. One easily arrives at $k_{2} \sim 0$ and (recall the definition $p=\epsilon \tilde{p}$ ) it follows that

$$
\frac{\alpha_{1}^{c}(0)}{2 \tilde{c}} \sim 0, \frac{\alpha_{1}^{s}(0)}{2 \tilde{c}} \sim-2\left(T_{0}-\frac{S_{0} T_{1}}{S_{1}}\right) \frac{1}{\log p+S} .
$$

The constant $S$ that occurs, which behaves like $\log \epsilon$ as $\epsilon$ vanishes, is given in Appendix C. As detailed there, we also have

$$
p \tilde{g}(p)=\frac{1}{\log p+S} \text { if } g(t)=v(s t)-\mathrm{e}^{s t}+1 \text { and } s=\mathrm{e}^{-S}
$$

where the function $v$ (Erdélyi et al. 1955, pp. 217-219) fulfils

$$
v(x)=\int_{0}^{\infty} \frac{x^{t} \mathrm{~d} t}{\Gamma(t+1)}, \quad v(0)=0, \quad v(x)-\mathrm{e}^{x} \rightarrow 0 \text { as } x \rightarrow \infty
$$

with $\Gamma$ the usual Gamma function. Accordingly, exploiting (3.3) and (4.11)-(4.12) the steady net forces $\boldsymbol{R}^{1}$ and $\boldsymbol{R}^{2}$ on the cylinders now become

$$
\frac{R_{x}^{n}}{4 \pi \mu \Omega a} \sim(-1)^{n}\left[d_{0}^{(0)}-\frac{S_{0}}{S_{1}} d_{0}^{(1)}\right]+\left[T_{0}-\frac{S_{0} T_{1}}{S_{1}}\right]\left[1-(-1)^{n}\left(d_{0}^{(2)}-\frac{S_{2}}{S_{1}} d_{0}^{(1)}\right)\right], \quad R_{y}^{n} \sim 0 .
$$

Hence, at the very first order of approximation, each cylinder $\mathscr{C}_{n}$ experiences a steady net force $\boldsymbol{R}^{n}$ normal to the line of centres (as in Watson 1995) and independent of the vanishing Reynolds number. Constructing a higher-order expansion would introduce a Reynolds-number dependence and a non-zero 'lift'. However, as noticed in Ueda et al. (2001) for the case of an impulsively translating and rotating circular cylinder, such a task would require taking into account nonlinear inertial effects, i.e. establishing a much more cumbersome approximation of the flow about the two-cylinder cluster. 

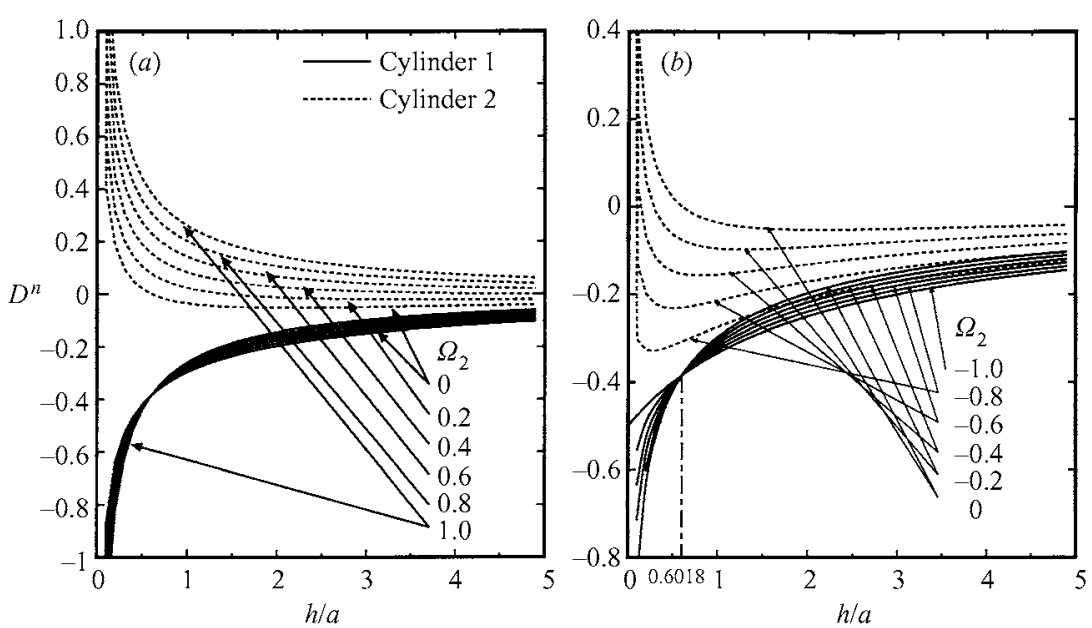

FIGURE 5. Drag coefficients $D^{1}$ (solid curves) and $D^{2}$ (dashed curves) versus the gap $h / a$ between identical cylinders $\left(a_{1}=a_{2}=a\right)$ for different values of $\Omega_{2}$ with $0 \leqslant\left|\Omega_{2}\right| \leqslant \Omega_{1}=1$. (a) Co-rotating cylinders $\left(\Omega_{2} \geqslant 0\right) ;(b)$ counter-rotating cylinders $\left(\Omega_{2} \leqslant 0\right)$.

From (4.29), the total steady force, $\boldsymbol{R}^{1}+\boldsymbol{R}^{2}$, exhibits the simple behaviour

$$
\boldsymbol{R}^{1}+\boldsymbol{R}^{2} \sim 8 \pi \mu \Omega a\left[T_{0}-\frac{S_{0} T_{1}}{S_{1}}\right] \boldsymbol{e}_{x} .
$$

We first present numerical results for two equal $\left(a_{1}=a_{2}=a\right)$ rotating cylinders $\mathscr{C}_{1}$ and $\mathscr{C}_{2}$ of arbitrary angular velocities $\Omega_{1}$ and $\Omega_{2}$. Selecting $\Omega_{2}=-\Omega_{1}$ will permit us to discuss Jeffery's paradox. If $a_{1}=a_{2}$ then $d_{0}^{(2)}=0$ and, see Watson (1995), $T_{1}=S_{2}=0$. Other coefficients $d_{0}^{(1)}, d_{0}^{(0)}, S_{0}, S_{1}$ and $T_{0}$ occurring in (4.29) are easily computed from (4.13)-(4.14) and Watson (1995). Omitting the details, one finally obtains

$$
\frac{R_{x}^{n}}{4 \pi \mu \Omega a}=(-1)^{n} \frac{d\left(\Omega_{1}+\Omega_{2}\right)}{\sinh 2 \alpha+2 \alpha}\left\{\frac{\sinh 2 \alpha}{2 \sinh ^{2} \alpha}-\frac{2 \alpha \sinh ^{2} \alpha}{S(\alpha)[\sinh 2 \alpha+2 \alpha]}\right\}+\frac{d\left(\Omega_{2}-\Omega_{1}\right)}{2 \sinh 2 \alpha}
$$

where $S(\alpha)$ is defined as in equation (42) of Watson (1995) and, by deducing from (4.17) that $2 a \cosh \alpha=2 a+h$,

$$
T_{0}-\frac{S_{0} T_{1}}{S_{1}}=\frac{R_{x}^{1}+R_{x}^{2}}{8 \pi \mu \Omega a}=\frac{d\left(\Omega_{2}-\Omega_{1}\right)}{2 \sinh 2 \alpha}=\frac{a\left(\Omega_{2}-\Omega_{1}\right)}{2(2+h / a)} .
$$

Inspection of (4.31) suggests the following remarks:

(i) Two co-rotating identical cylinders of equal angular velocities $\left(\Omega_{2}=\Omega_{1}\right)$ experience opposite 'drag' forces which, as the reader may check, agree perfectly with the results of Watson (1995).

(ii) Two counter-rotating identical cylinders of opposite angular velocities $\left(\Omega_{2}=\right.$ $\left.-\Omega_{1}\right)$, as sketched in figure $1(b)$ and considered in detail by Jeffery (1922) in illustrating his paradox, experience the same and very simple 'drag' (use (4.32)).

In general $R_{x}^{1}$ and $R_{x}^{2}$ are different in magnitude and for symmetry reasons we confine ourselves to the choice $0 \leqslant\left|\Omega_{2}\right| \leqslant \Omega_{1}=1$. Figure $5(a, b)$ shows the 'drag' coefficients $D^{n}=R_{x}^{n} /[4 \pi \mu \Omega a]$ versus the gap variable $h / a$ both for co-rotating and counter-rotating identical cylinders. By virtue of (4.31)-(4.32), the cluster 'drag' 

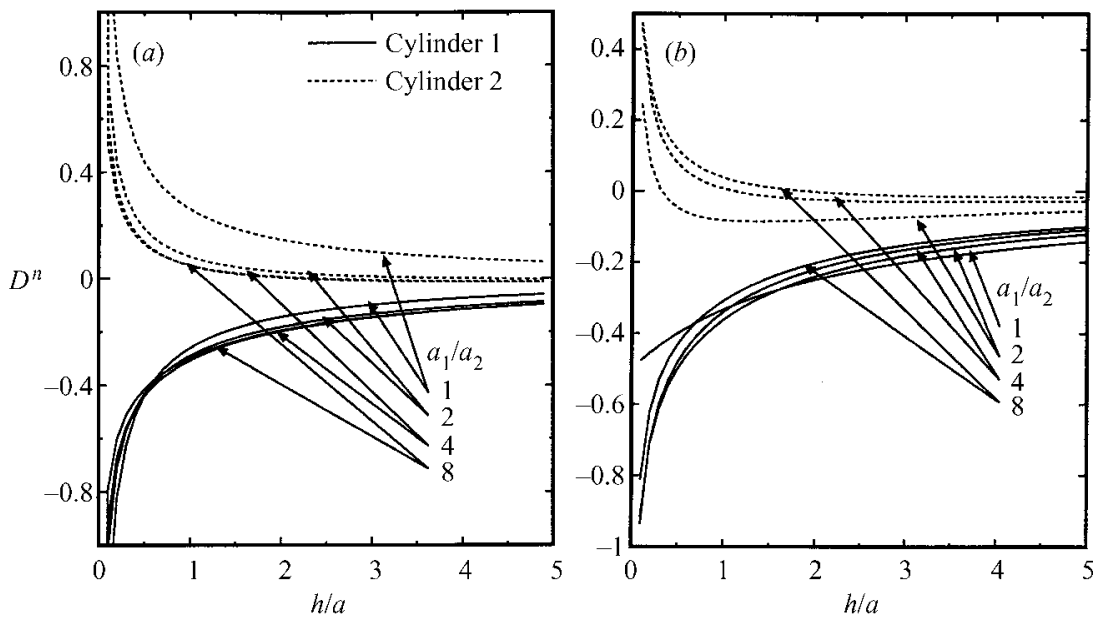

FiguRE 6. Drag coefficients $D^{1}$ (solid curves) and $D^{2}$ (dashed curves) versus the gap $h / a$ between cylinders of equal or opposite angular velocities for different values of $a_{2}$ with $a_{2}<a_{1}=a$. (a) Co-rotating cylinders $\left(\Omega_{2}=\Omega_{1}=1\right)$; $(b)$ counter-rotating cylinders $\left(\Omega_{2}=-\Omega_{1}=-1\right)$.

coefficient $D^{1}+D^{2}$ is proportional to the angular velocity difference $\Omega_{2}-\Omega_{1}$ and it is finite in the whole range $h / a \geqslant 0$, i.e. even for nearly touching cylinders. For Jeffery's conditions $\left(\Omega_{2}=-\Omega_{1}=-1\right)$ this remains true for $D^{1}=D^{2}$ as depicted in figure $5(b)$ : each cylinder experiences the same negative and finite drag coefficient which asymptotes to $-a / 2$ as $h / a$ vanishes. However, since $\alpha \sim(h / a)^{1 / 2}$ as $h \rightarrow 0$ with $S(\alpha)$ of order of $\alpha^{2}$, each drag coefficient $D^{n}$ diverges as our identical cylinders approach each other if $\Omega_{1}+\Omega_{2} \neq 0$. Indeed, one immediately obtains

$$
D^{n}=\frac{R_{x}^{n}}{4 \pi \mu \Omega a} \sim(-1)^{n}\left(\Omega_{1}+\Omega_{2}\right) \frac{a}{4}\left(\frac{a}{h}\right)^{1 / 2} \text { as } h \rightarrow 0 .
$$

For $\Omega_{1}=1$ note that $D^{1}$ is negative and decreases in magnitude as $h / a$ increases whatever the sign of $\Omega_{2}$. In addition, $-D^{1}$ is seen to decrease or increase with $\Omega_{2}$ for $h / a$ greater or lower respectively than a critical value $h_{c} / a \sim 0.6018$ of the gap between the cylinders. In contrast, the drag coefficient $D^{2}$ exhibits more subtle trends: for any value of $h / a$ it increases with $\Omega_{2}$ and if it is positive for $\Omega_{2}$ roughly exceeding 0.4 , then for other values of the angular velocity $\Omega_{2} \neq-1$ it is positive for sufficiently close cylinders and negative for sufficiently distant cylinders. If $\Omega_{2} \rightarrow-\Omega_{1}=-1$ the coefficient $D^{2}$ collapses to $D^{1}$, as previously pointed out. Finally, since $\Omega_{1}$ is unchanged, note that $D^{2}$ is more sensitive than $D^{1}$ to changes in $\Omega_{2}$ for a given geometry ( $h$ prescribed), especially for sufficiently close cylinders.

Figure $6(a, b)$ addresses size effects for non-identical cylinders with $0<a_{2}<a_{1}=a$ for $\Omega_{2}=\Omega_{1}=1$ in figure 6(a) and for $\Omega_{2}=-\Omega_{1}=-1$ in figure 6(b). Each coefficient $S_{0}, S_{1}$ and $S_{2}=-T_{1}$ required is computed with reference to Watson $(1995,1996)$. Again, $-D^{1}$ is positive and decreases as $h / a$ increases whereas $D^{2}$ is either positive or negative and may either decrease or increase with $h / a$. The larger cylinder $\mathscr{C}_{1}$ always experiences the greater drag, i.e. $-D^{1}>\left|D^{2}\right|$, as revealed by figures $6(a)$ and $6(b)$. Note that $D^{2}$ decreases or increases as $a_{2} / a_{1}$ decreases for co-rotating or counter-rotating cylinders respectively whereas the behaviour of $-D^{1}$ depends upon the gap between the cylinders. Any computed drag coefficient $D^{n}$ is again seen to 

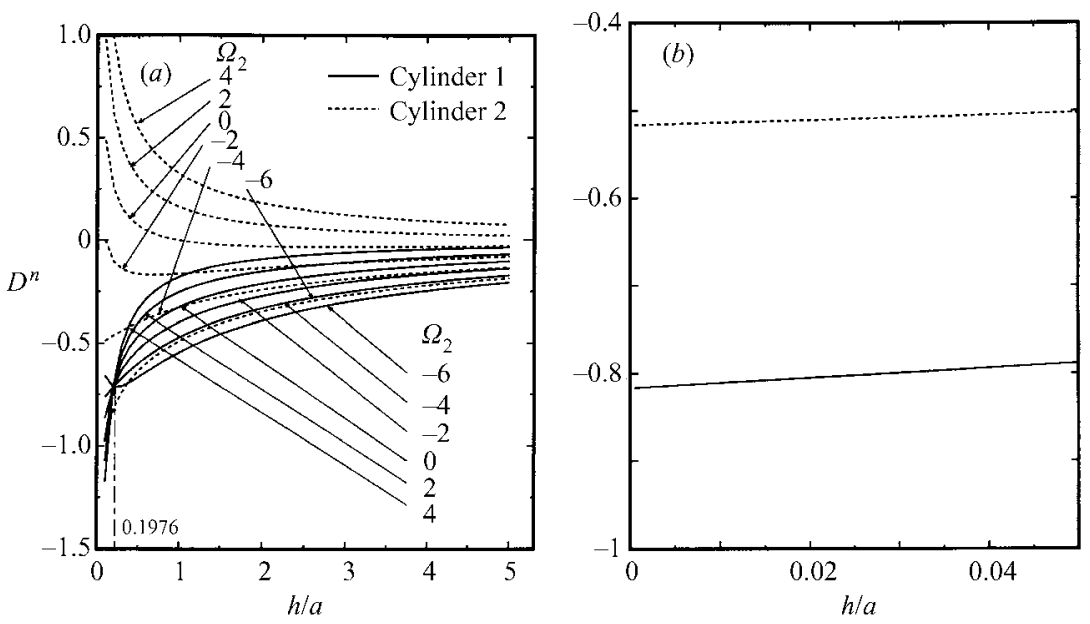

FIGURE 7. Drag coefficients $D^{1}$ (solid curves) and $D^{2}$ (dashed curves) versus the gap $h / a$ between non-identical cylinders of unequal angular velocities for $a_{1}=2 a_{2}=a$ and $\Omega_{1}=1$. (a) Computed coefficients for different values of $\Omega_{2}$, including the critical value $\Omega_{2 c}=-4$. (b) Magnification, for a vanishing gap between the counter-rotating cylinders, of the drag coefficient curves at the critical angular velocity $\Omega_{2 c}=-4$.

diverge as $h / a$ vanishes. One immediately obtains

$$
\eta \sim \frac{2 a_{2}}{a_{1}\left(a_{1}+a_{2}\right)} h=e h, \quad \alpha \sim(e h)^{1 / 2}, \quad \beta \sim \frac{a_{1}}{a_{2}}(e h)^{1 / 2} \text { as } h \rightarrow 0,
$$

and letting $h$ tend to zero in (4.29) suggests distinguishing the following cases:

(i) Case 1. In general $a_{1}^{2} \Omega_{1}+a_{2}^{2} \Omega_{2} \neq 0$ and in such circumstances one arrives at

$$
\frac{R_{x}^{n}}{4 \pi \mu \Omega a} \sim(-1)^{n} a_{2} \sqrt{\frac{a_{1}\left(a_{1}+a_{2}\right)}{2 a_{2}}} \frac{\left[a_{1}^{2} \Omega_{1}+a_{2}^{2} \Omega_{2}\right]}{\left(a_{1}+a_{2}\right)^{2}} \frac{1}{\sqrt{h}} \text { as } h \rightarrow 0 .
$$

Figure $6(a, b)$, since $a_{1}>a_{2}$, is one such case.

(ii) Case 2. If it appears that $a_{1}^{2} \Omega_{1}+a_{2}^{2} \Omega_{2}=0$ the previous diverging expansion (4.35) vanishes and a higher-order analysis is needed. One obtains

$$
d_{0}^{(0)}=0, \quad d_{0}^{(2)} \sim d_{0}^{(2)}, \quad T_{0} \sim T_{0}^{\prime}, \quad d_{0}^{(1)} \sim d_{0}^{\prime(1)} h^{1 / 2}, \quad S_{2} \sim S_{2}^{\prime} h^{1 / 2}, \quad S_{1} \sim S_{1}^{\prime} h
$$

with primed quantities of order of unity. Accordingly, each drag coefficient asymptotes to a finite value as the cylinders approach with

$$
\frac{R_{x}^{n}}{4 \pi \mu \Omega a} \sim T_{0}^{\prime}\left[1-(-1)^{n}\left(d_{0}^{\prime(2)}-\frac{S_{2}^{\prime}}{S_{1}^{\prime}} d_{0}^{\prime(1)}\right)\right] .
$$

Although it is easy to establish that

$$
\begin{gathered}
d_{0}^{\prime(1)}=-\frac{4 a_{2} a_{1}^{2}}{\left(a_{1}+a_{2}\right)^{3}}\left[\frac{2 a_{2}}{a_{1}\left(a_{1}+a_{2}\right)}\right]^{1 / 2}, d_{0}^{\prime(2)}=\frac{\left(a_{1}-a_{2}\right)}{\left(a_{1}+a_{2}\right)^{3}}\left(a_{1}^{2}+a_{2}^{2}-4 a_{1} a_{2}\right), \\
T_{0}^{\prime}=\frac{2 a_{1}^{2} a_{2}^{2}\left(\Omega_{2}-\Omega_{1}\right)+\left(a_{1}-a_{2}\right)\left(a_{2}^{3} \Omega_{2}+a_{1}^{3} \Omega_{1}\right)}{\left(a_{1}+a_{2}\right)^{3}}
\end{gathered}
$$

obtaining $S_{1}^{\prime}, S_{2}^{\prime}$ is much more involved and it has not been possible to obtain these coefficients in closed form. The occurrence of Case 2, previously encountered in 
figure 5(b) when $a_{2}=a_{1}$, suggests plotting the drag coefficients $D^{n}$ for $a_{2}<a_{1}=$ $a, \Omega_{1}=1$ and a few values of $\Omega_{2}$, including the critical angular velocity $\Omega_{2 c}=-1 / a_{2}^{2}$. The results obtained are depicted in figure $7(a, b)$ for $a_{2}=1 /(2 a)$. As illustrated in figure 7(a), for $h / a$ roughly exceeding $1 / 4$ both $-D^{1}$ and $\operatorname{sgn}\left(\Omega_{2}\right) D^{2}$ are positive and decrease as $h / a$ increases. As theoretically predicted, $D^{1}$ and $D^{2}$ diverge for nearly touching cylinders if $\Omega_{2} \neq \Omega_{2 c}$ with $(-1)^{n} D^{n}\left(\Omega_{2}-\Omega_{2 c}\right)>0$. For the critical angular velocity $\Omega_{2 c}=-4$, the coefficients $D^{1}$ and $D^{2}$ become finite when $h / a$ vanishes, as clearly seen from the magnified plot of figure $7(b)$. In contrast to the case of equal cylinders in figure $5(b)$, we obtain different values of $D^{1}$ and $D^{2}$ for very close cylinders.

\section{Conclusions}

A theoretical analysis has been developed to obtain the long-time viscous flow past an arbitrary $\mathrm{N}$-cylinder cluster made up of sufficiently close and either rotating circular cylinders or arbitrarily shaped but motionless cylinders. The procedure advocated, which matches an inner steady Stokes approximation with an outer unsteady Oseen solution by exploiting integral representations of the stream function and the vorticity, extends the previous works of Nakanishi et al. (1997) and Ueda et al. (2001) which dealt with a single translating and rotating circular cylinder.

The solution has been analytically worked out for two rotating circular cylinders, a simple geometry of interest for the Jeffery paradox. The steady flow past the cluster is then obtained by letting time tend to infinity for a non-zero external flow. The firstorder approximation derived then agrees with Watson (1996) and it extends the results of Lee \& Leal (1986) to the case of rotating cylinders. The motivating case of the viscous flow, both steady and quiescent far from the cluster, is adequately addressed by making the external flow vanish before letting time tend to infinity. The first-order approximations of the lift and drag (parallel and normal to the line of centres) force coefficients obtained are zero and independent of the vanishing Reynolds number respectively. The sensitivity of the computed drag coefficients to the angular velocities, radii and gap between the cylinders is discussed. Whereas the total drag experienced by the cluster never diverges, each drag coefficient remains finite or increases in magnitude as $h^{-1 / 2}$ if the gap $h$ between cylinders vanishes for $a_{1}^{2} \Omega_{1}+a_{2}^{2} \Omega_{2}=0$ and $a_{1}^{2} \Omega_{1}+a_{2}^{2} \Omega_{2} \neq 0$ respectively. Finally, we note that a higher-order approximation is needed in order to predict the lift force on each rotating cylinder. Unfortunately, such a refined analysis deals with nonlinear terms and becomes very involved.

The authors gratefully acknowledge the financial support provided by the Japan Society for the Promotion of Science (JSPS) under its Grant-in-Aid for Scientific Research number C12650178 and express their warm thanks to Professor Huerre for his help in preparing the manuscript. Finally, special thanks are addressed to one anonymous referee who kindly drew the author's attention to the paper by Stokes (1850).

\section{Appendix A. Approximation of $I\left(\zeta^{\prime}\right)$ and $I\left(\psi^{\prime}\right)$}

This Appendix details, for $I\left(\zeta^{\prime}\right)$, the method used in approximating both $I\left(\zeta^{\prime}\right)$ and $I\left(\psi^{\prime}\right)$. First, we recall that $f_{o}^{\prime}(\boldsymbol{Y})=f^{\prime}(\boldsymbol{Y} / \epsilon)$ in both the outer and the intermediate domains, i.e. say for $|\boldsymbol{Y}| \geqslant \epsilon / \delta$ with $\epsilon \ll \delta \ll 1$. If we introduce the domains $\mathscr{D}_{1}=\left\{\boldsymbol{y} \in \mathscr{D} ;|\boldsymbol{y}|<\delta^{-1}\right\}$ and $\mathscr{D}_{2}=\{\boldsymbol{Y} ;|\boldsymbol{Y}| \geqslant \epsilon / \delta\}$ such that, under the assumption 
$h=O(1), \mathscr{D}=\mathscr{D}_{1} \cup \mathscr{D}_{2}$ and apply the change of scale $\boldsymbol{Y}=\epsilon \boldsymbol{y}$ to the integration over $\mathscr{D}_{2}$, we obtain

$$
\begin{gathered}
-\frac{\pi}{\epsilon} I\left(\zeta^{\prime}\right)=\int_{\mathscr{D}} f^{\prime}(\boldsymbol{y}) K_{0}(\tilde{c}|\boldsymbol{X}-\epsilon \boldsymbol{y}|) \mathrm{d} S(\boldsymbol{y})=T_{1}(\boldsymbol{X})+T_{2}(\boldsymbol{X}) / \epsilon^{2}, \\
T_{1}(\boldsymbol{X})=\int_{\mathscr{D}_{1}} f^{\prime}(\boldsymbol{y}) K_{0}(\tilde{c}|\boldsymbol{X}-\epsilon \boldsymbol{y}|) \mathrm{d} S(\boldsymbol{y}), T_{2}(\boldsymbol{X})=\int_{\mathscr{D}_{2}} f_{o}^{\prime}(\boldsymbol{Y}) K_{0}(\tilde{c}|\boldsymbol{X}-\boldsymbol{Y}|) \mathrm{d} S(\boldsymbol{Y}) .
\end{gathered}
$$

Since $R=|\boldsymbol{X}|=O(1) \gg \epsilon|\boldsymbol{y}|$ for $\boldsymbol{y}$ in $\mathscr{D}_{1}$ (use $\epsilon \ll \delta$ ), the expansion (3.15) yields

$$
T_{1}(\boldsymbol{X})=H_{0}^{1}(\epsilon, \delta) K_{0}(\tilde{c} R)+\sum_{m \geqslant 1}\left[H_{m}^{1, c}(\epsilon, \delta) \cos m \theta+H_{m}^{1, s}(\epsilon, \delta) \sin m \theta\right] K_{m}(\tilde{c} R)
$$

where the presence of the real coefficients $H_{0}^{1}, H_{m}^{1, c}$ and $H_{m}^{1, s}$ is dictated by the inner representation $f_{i}^{\prime}$ (which holds within the whole domain $\mathscr{D}_{1}$ ). They are defined as

$$
\begin{aligned}
H_{0}^{1}(\epsilon, \delta) & =\int_{\mathscr{D}_{1}} f^{\prime}(\boldsymbol{y}) I_{0}(\epsilon \tilde{c}|\boldsymbol{y}|) \mathrm{d} S(\boldsymbol{y}), \\
H_{m}^{1, c}(\epsilon, \delta)+\mathrm{i} H_{m}^{1, s}(\epsilon, \delta) & =2 \int_{\mathscr{D}_{1}} f^{\prime}(\boldsymbol{y}) I_{m}(\epsilon \tilde{c}|\boldsymbol{y}|) \mathrm{e}^{\mathrm{i} m \varphi} \mathrm{d} S(\boldsymbol{y}), \quad m \geqslant 1 .
\end{aligned}
$$

Assuming that there exist two positive integers $K$ and $L$ and real functions $f_{l}^{\prime k}(\varphi)$, at most of the order of $\bar{f}_{o}^{\prime}$ for integers $l \geqslant-L$ and zero otherwise, such that the outer representation $f_{o}^{\prime}(\boldsymbol{Y})$ admits, for $O(\epsilon / \delta) \leqslant|\boldsymbol{Y}| \ll 1$ and $\varphi=\arg (\boldsymbol{Y})$, the expansion

$$
f_{o}^{\prime}(\boldsymbol{Y})=f_{0}^{\prime}(\boldsymbol{Y})+\sum_{n \geqslant 1}\left[f_{n}^{\prime}(\boldsymbol{Y})|\boldsymbol{Y}|^{n}+f_{-n}^{\prime}(\boldsymbol{Y})|\boldsymbol{Y}|^{-n}\right], \quad f_{l}(\boldsymbol{Y})=\sum_{k=0}^{K} f_{l}^{\prime k}(\varphi) \log ^{k}|\boldsymbol{Y}|,
$$

permits us to define $f_{o}^{\prime}(\boldsymbol{Y})$ within $\mathscr{D}_{3}=\mathbb{R}^{2} \backslash \mathscr{D}_{2}$ (except at the origin) and to write

$$
\begin{gathered}
T_{2}(\boldsymbol{X})+T_{3}(\boldsymbol{X})=\operatorname{pf} \int_{\mathbb{R}^{2}} f_{o}^{\prime}(\boldsymbol{Y}) K_{0}(\tilde{c}|\boldsymbol{X}-\boldsymbol{Y}|) \mathrm{d} S(\boldsymbol{Y}), \\
T_{3}(\boldsymbol{X})=\operatorname{pf} \int_{\mathscr{D}_{3}} f_{o}^{\prime}(\boldsymbol{Y}) K_{0}(\tilde{c}|\boldsymbol{X}-\boldsymbol{Y}|) \mathrm{d} S(\boldsymbol{Y}),
\end{gathered}
$$

where the symbol pf denotes the integration in the finite part sense of Hadamard (1932). For further details the reader is referred to Sellier (1997). Since $|\boldsymbol{Y}|<\epsilon / \delta<$ $|\boldsymbol{X}|=O(1)$ for $\boldsymbol{Y}$ in $\mathscr{D}_{3}$, we again invoke (3.15), together with (3.19), to cast $T_{3}(\boldsymbol{X})$ into the same expansion as (A 3) but with real coefficients $H_{0}^{3}(\epsilon, \delta), H_{0}^{3, c}(\epsilon, \delta)$ and $H_{0}^{3, s}(\epsilon, \delta)$ such that

$$
\begin{aligned}
H_{0}^{3}(\epsilon, \delta) & =\operatorname{pf} \int_{\mathscr{D}_{3}} f_{o}^{\prime}(\boldsymbol{Y}) I_{0}(\tilde{c}|\boldsymbol{Y}|) \mathrm{d} S(\boldsymbol{Y}), \\
H_{m}^{3, c}(\epsilon, \delta)+\mathrm{i} H_{m}^{3, s}(\epsilon, \delta) & =2 \operatorname{pf} \int_{\mathscr{D}_{3}} f_{o}^{\prime}(\boldsymbol{Y}) I_{m}(\tilde{c}|\boldsymbol{Y}|) \mathrm{e}^{\mathrm{i} m \varphi} \mathrm{d} S(\boldsymbol{Y}), \quad m \geqslant 1 .
\end{aligned}
$$

Finally, our results (A 1), (A 3) and (A 7)-(A 8), in conjunction with (3.16), yield the expansion (3.20) under the definitions

$$
A_{0}(\epsilon)=\frac{\epsilon}{\pi}\left(H_{0}^{1}-\frac{H_{0}^{3}}{\epsilon^{2}}\right), \quad A_{m}^{c}(\epsilon)=\frac{\epsilon}{\pi}\left(H_{m}^{1, c}-\frac{H_{m}^{3, c}}{\epsilon^{2}}\right), \quad A_{m}^{s}(\epsilon)=\frac{\epsilon}{\pi}\left(H_{m}^{1, s}-\frac{H_{m}^{3, s}}{\epsilon^{2}}\right) .
$$


Since $f_{o}^{\prime}(\epsilon \boldsymbol{y})=f^{\prime}(\boldsymbol{y})$ in the intermediate domain, the reader may easily check that the above coefficients $A_{0}, A_{m}^{c}$ and $A_{m}^{s}$ indeed do not depend upon $\delta$. Finally, employing the decomposition (3.11) and assuming for the function $g_{o}^{\prime}(\boldsymbol{Y})=\zeta_{o}^{\prime}(\boldsymbol{Y}) \exp (\boldsymbol{W} \cdot \boldsymbol{Y})$ the following counterpart of (A 6), for $O(\epsilon / \delta) \leqslant|\boldsymbol{Y}| \ll 1$ and other positive integers $K^{\prime}$ and $L^{\prime}$ :

$$
g_{o}^{\prime}(\boldsymbol{Y})=g_{0}^{\prime}(\boldsymbol{Y})+\sum_{n \geqslant 1}\left[g_{n}^{\prime}(\boldsymbol{Y})|\boldsymbol{Y}|^{n}+g_{-n}^{\prime}(\boldsymbol{Y})|\boldsymbol{Y}|^{-n}\right], \quad g_{l}(\boldsymbol{Y})=\sum_{k=0}^{K^{\prime}} g_{l}^{\prime k}(\varphi) \log ^{k}|\boldsymbol{Y}|,
$$

one expands the integral $I\left(\psi^{\prime}\right)$ in a similar fashion and, by virtue of (3.12), obtains the decomposition (3.21).

\section{Appendix B. Matching of $\zeta^{\prime}$ and $\psi^{\prime}$}

In this Appendix we derive the key approximations (3.28)-(3.29) and the far-field behaviour (3.31). By virtue of (3.11), the inner expansion (3.26), rewritten in outer variables $(R, \theta)$ for $|\boldsymbol{x}| \rightarrow \infty$, becomes

$$
\begin{array}{r}
\zeta_{i}^{\prime}=-\sum_{m \geqslant 1}\left[E_{m}^{c}(\epsilon) \cos m \theta+E_{m}^{s}(\epsilon) \sin m \theta\right] \epsilon^{-m} R^{m}-\left[\gamma+\log \left(\frac{1}{2} \tilde{c}\right)+\log R\right] \alpha_{0}(0) \\
-E_{0}(\epsilon)+\sum_{m \geqslant 1}\left[F_{m}^{c}(\epsilon) \cos m \theta+F_{m}^{s}(\epsilon) \sin m \theta\right] \epsilon^{m} R^{-m}
\end{array}
$$

with real coefficients $\alpha_{0}(0), E_{m}^{c}(\epsilon), E_{m}^{s}(\epsilon), F_{m}^{c}(\epsilon)$ and $F_{m}^{s}(\epsilon)$ of order of $\bar{\zeta}_{i}^{\prime}$ and

$F_{m}^{c}(\epsilon)+\mathrm{i} F_{m}^{s}(\epsilon)=\frac{1}{2 \pi m} \int_{\mathscr{C}}\left\{\rho^{m} \mathrm{e}^{\mathrm{i} m \varphi} \frac{\partial \zeta^{\prime}}{\partial n}(\boldsymbol{y})-\zeta^{\prime}(\boldsymbol{y}) \frac{\partial}{\partial n}\left[\rho^{m} \mathrm{e}^{\mathrm{i} m \varphi}\right]\right\} \mathrm{d} l(\boldsymbol{y})$ for $m \geqslant 1$.

Neglecting, as explained in $\S 3.3$, the integral on the right-hand side of (3.20) we also have

$$
\begin{aligned}
\zeta_{o}^{\prime}=[ & \left.\alpha_{0}(\epsilon)-A_{0}(\epsilon)\right] K_{0}(\tilde{c} R) \\
& +\sum_{m \geqslant 1}\left\{\left[\epsilon^{m} \alpha_{m}^{c}(\epsilon)-A_{m}^{c}(\epsilon)\right] \cos m \theta+\left[\epsilon^{m} \alpha_{m}^{s}(\epsilon)-A_{m}^{s}(\epsilon)\right] \sin m \theta\right\} K_{m}(\tilde{c} R)
\end{aligned}
$$

with retained coefficients $\alpha_{0}(\epsilon)-A_{0}(\epsilon), \epsilon^{m} \alpha_{m}^{c}(\epsilon)-A_{m}^{c}(\epsilon)$ and $\epsilon^{m} \alpha_{m}^{s}(\epsilon)-A_{m}^{s}(\epsilon)$ of order of $\bar{\zeta}_{o}^{\prime}$. Using (B 3) we now expand $\zeta_{o}^{\prime}$ as $R \rightarrow 0$ by employing the asymptotic approximations (Abramowitz \& Stegun 1965, p. 375)

$$
K_{0}(u)=-[\gamma+\log (u / 2)]+O\left(u^{2} \log u\right), \quad K_{m}(u) \sim \frac{(m-1) !}{2}\left(\frac{2}{u}\right)^{m} \text { as } u \rightarrow 0
$$

where $\gamma$ denotes Euler's constant. According to the matching principle (Van Dyke 1974, p. 90) the expansion derived must match with (B 1). Thus, $E_{m}^{c}(\epsilon)=E_{m}^{s}(\epsilon)=0$ and one deduces the far-field behaviour (3.27) and, as explained in $\S 3.4$, that $\alpha_{0}(0)=0$. Finally, matching the constant terms yields, at the retained order of approximation, $E_{0}(\epsilon)=0, \alpha_{0}(\epsilon)-A_{0}(\epsilon)=0$ and

$$
\epsilon \alpha_{1}^{c}-A_{1}^{c}=\epsilon \tilde{c} F_{1}^{c}=\epsilon \alpha_{1}^{c}(0), \epsilon \alpha_{1}^{s}-A_{1}^{c}=\epsilon \tilde{c} F_{1}^{s}=\epsilon \alpha_{1}^{s}(0), \epsilon^{m} \alpha_{1}^{c, s}-A_{m}^{c, s}=0 \text { if } m \geqslant 2 .
$$

Using these results and (3.26) one immediately obtains (3.28)-(3.29). Let us now turn to the matching of $\psi^{\prime}$. Setting $g_{i}^{\prime}(\boldsymbol{y})=\zeta_{i}^{\prime}(\boldsymbol{y}) \exp (\epsilon \boldsymbol{W} \cdot \boldsymbol{y})$ and denoting by $U_{o}(\boldsymbol{X})$ and 
$U_{i}(\boldsymbol{x})$ the integrals arising on the right-hand sides of (3.21)-(3.23), the use of (3.11) yields

$$
\begin{gathered}
\left.-2 \pi U_{i}(\boldsymbol{x})=\operatorname{pf} \int_{\mathscr{D}} g_{i}^{\prime}(\boldsymbol{y})\left\{\log r-\sum_{m \geqslant 1} \frac{1}{m}\left(\frac{\rho}{r}\right)^{m} \cos [m(\theta-\varphi)]\right\} \mathrm{d} S(\boldsymbol{y})+T_{i}(\boldsymbol{x}), \quad \text { (B } 6\right) \\
-\frac{2 U_{o}(\boldsymbol{X})}{\left(\pi \epsilon^{2}\right)^{-1}}=\operatorname{pf} \int_{\mathbb{R}^{2}} g_{o}^{\prime}(\boldsymbol{Y})\left\{\log |\boldsymbol{Y}|-\sum_{m \geqslant 1} \frac{1}{m}\left(\frac{R}{|\boldsymbol{Y}|}\right)^{m} \cos [m(\theta-\varphi)]\right\} \mathrm{d} S(\boldsymbol{Y})+\frac{T_{o}(\boldsymbol{X})}{\epsilon^{-2}}, \\
T_{i}(\boldsymbol{x})=\operatorname{pf} \int_{\rho>r} g_{i}^{\prime}(\boldsymbol{y})\left\{\log \left(\frac{\rho}{r}\right)-\sum_{m \geqslant 1} \frac{1}{m}\left[\left(\frac{r}{\rho}\right)^{m}-\left(\frac{\rho}{r}\right)^{m}\right] \cos [m(\theta-\varphi)]\right\} \mathrm{d} S(\boldsymbol{y}), \\
\epsilon^{2} T_{o}(\boldsymbol{X})=\operatorname{pf} \int_{|\boldsymbol{Y}|<R} g_{o}^{\prime}(\boldsymbol{Y})\left\{\log \left(\frac{R}{|\boldsymbol{Y}|}\right)^{m}(\mathrm{~B} 8)\right. \\
\left.-\sum_{m \geqslant 1} \frac{1}{m}\left[\left(\frac{|\boldsymbol{Y}|}{R}\right)^{m}-\left(\frac{R}{|\boldsymbol{Y}|}\right)^{m}\right] \cos [m(\theta-\varphi)]\right\} \mathrm{d} S(\boldsymbol{Y})
\end{gathered}
$$

Due to the existence of the overlap domain and the definition of $g_{i}^{\prime}$ and $g_{o}^{\prime}$, we have, as $|\boldsymbol{y}|=O\left(\delta^{-1}\right)$ and $|\boldsymbol{Y}|=O(\epsilon / \delta)$,

$$
g_{i}^{\prime}(\boldsymbol{y})=g_{o}^{\prime}(\boldsymbol{Y}) \sim \zeta_{o}^{\prime}(\boldsymbol{Y})=\epsilon a(\varphi) K_{1}(\tilde{c}|\boldsymbol{Y}|), \quad a(\varphi)=\alpha_{1}^{c}(0) \cos \varphi+\alpha_{1}^{s}(0) \sin \varphi .
$$

In addition (Abramowitz \& Stegun 1965, p. 375) note that

$$
K_{1}(u)=\frac{1}{u}+F(u), \quad F(u)=\sum_{k=0}^{\infty}\left(a_{k}-b_{k} \log u\right) u^{2 k+1}
$$

Accordingly, as $|\boldsymbol{x}| \rightarrow 0$ and $|\boldsymbol{X}| \rightarrow \infty$, we obtain

$$
\begin{array}{r}
T_{i}(\boldsymbol{x})=-\frac{\pi a(\theta)}{\tilde{c}}\left\{\mathrm{pf} \int_{\rho>r}\left(\frac{r}{\rho}-\frac{\rho}{r}\right) \mathrm{d} \rho+\epsilon \tilde{c} \mathrm{pf} \int_{\rho>r} F(\tilde{c} \epsilon \rho)\left(r-\frac{\rho^{2}}{r}\right) \mathrm{d} \rho\right\}, \\
T_{o}(\boldsymbol{X})=-\frac{\pi a(\theta)}{\epsilon \tilde{c}}\left\{\mathrm{pf} \int_{0}^{R}\left(\frac{t}{R}-\frac{R}{t}\right) \mathrm{d} t+\tilde{c} \int_{0}^{R} F(\tilde{c} t)\left(\frac{t}{R}-\frac{R}{t}\right) t \mathrm{~d} t\right\} .
\end{array}
$$

Using changes of scale $\rho=r t^{\prime}$ and $t=\epsilon \rho$ for the last integrals on the right-hand sides of (B 12)-(B 13) and the key relation pf $\int_{0}^{\infty} x^{m} \log ^{n} x \mathrm{~d} x=0$ for any positive integers $m$ and $n$ (Sellier 1994), one deduces from expansion (B 11) that

$$
\begin{aligned}
J & =\operatorname{pf} \int_{\rho>r} F(\tilde{c} \epsilon \rho)\left(r-\frac{\rho^{2}}{r}\right) \mathrm{d} \rho=\int_{0}^{R} \frac{F(\tilde{c} t)}{\epsilon^{2}}\left(\frac{t}{R}-\frac{R}{t}\right) t \mathrm{~d} t \\
& =r^{2} \operatorname{pf} \int_{1}^{\infty} \frac{F\left(\tilde{c} \epsilon r t^{\prime}\right)}{\left(1-t^{\prime 2}\right)^{-1}} \mathrm{~d} t^{\prime} .
\end{aligned}
$$

Through a straightforward calculation it follows that

$$
T_{i}(\boldsymbol{x})=-\frac{\pi a(\theta)}{\tilde{c}}\left\{\frac{r}{2}-r \log r+\epsilon \tilde{c} J\right\}, \quad T_{o}(\boldsymbol{X})=-\frac{\pi a(\theta)}{\epsilon \tilde{c}}\left\{\frac{R}{2}-R \log R+\epsilon^{2} \tilde{c} J\right\} .
$$


Using expansions (3.21)-(3.23) and the above results we thus deduce the following behaviours in the intermediate domain

$$
\begin{aligned}
\psi_{i}^{\prime}(\boldsymbol{x})= & -D_{0}(\epsilon)-\sum_{m \geqslant 1}\left[D_{m}^{c}(\epsilon) \cos m \theta+D_{m}^{s}(\epsilon) \sin m \theta\right] \epsilon^{-m} R^{m}+\sum_{m \geqslant 1}\left[G_{m}^{c}(\epsilon) \cos m \theta\right. \\
& \left.+G_{m}^{s}(\epsilon) \sin m \theta\right] \epsilon^{m} R^{-m}-\beta_{0} \log \left(\frac{R}{\epsilon}\right)+\frac{a(\theta)}{2 \tilde{c}}\left\{\frac{R}{2 \epsilon}-\frac{R}{\epsilon} \log \left(\frac{R}{\epsilon}\right)+\epsilon \tilde{c} J\right\} \\
& -\frac{1}{2 \pi} \mathrm{pf} \int_{\mathscr{D}} g_{i}^{\prime}(\boldsymbol{y})\left\{\log \left(\frac{R}{\epsilon}\right)-\sum_{m \geqslant 1} \frac{\epsilon^{m}}{m}\left(\frac{\rho}{R}\right)^{m} \cos [m(\theta-\varphi)]\right\} \mathrm{d} S(\boldsymbol{y})
\end{aligned}
$$

where the real coefficients $G_{m}^{c}$ and $G_{m}^{s}$ obey the definition (B 2) with $\zeta^{\prime}$ replaced by $\psi^{\prime}$ and

$$
\begin{aligned}
\psi_{o}^{\prime}(\boldsymbol{X})= & -\sum_{m \geqslant 1}\left\{\left[\epsilon^{m} \beta_{m}^{c}(\epsilon)+C_{m}^{c}(\epsilon)\right] \cos m \theta+\left[\epsilon^{m} \beta_{m}^{s}(\epsilon)+C_{m}^{s}(\epsilon)\right] \sin m \theta\right\} R^{-m} \\
& -C_{0}^{\prime}(\epsilon)-\left[\beta_{0}+C_{0}(\epsilon)\right] \log R+\frac{a(\theta)}{2 \tilde{c} \epsilon}\left\{\frac{1}{2} R-R \log R+\epsilon^{2} \tilde{c} J\right\} \\
& -\frac{1}{2 \pi \epsilon^{2}} \mathrm{pf} \int_{\mathbb{R}^{2}} g_{o}^{\prime}(\boldsymbol{Y})\left\{\log |\boldsymbol{Y}|-\sum_{m \geqslant 1} \frac{1}{m}\left(\frac{R}{|\boldsymbol{Y}|}\right)^{m} \cos [m(\theta-\varphi)]\right\} \mathrm{d} S(\boldsymbol{Y}) .
\end{aligned}
$$

For conciseness, we do not reproduce here the detailed matching of (B 16) and (B 17). Let us only say that $D_{1}^{c, s}(\epsilon)=O\left(\alpha_{1}^{c, s}\right)$ because we obtain

$$
D_{1}^{c}(\epsilon)+\mathrm{i} D_{1}^{s}(\epsilon)=\frac{\log \epsilon}{2 \tilde{c}}\left[\alpha_{1}^{c}(0)+\mathrm{i} \alpha_{1}^{s}(0)\right]-\frac{1}{2 \pi \epsilon} \operatorname{pf} \int_{\mathbb{R}^{2}} \frac{g_{o}^{\prime}(\boldsymbol{Y})}{|\boldsymbol{Y}|} \mathrm{e}^{\mathrm{i} m \varphi} \mathrm{d} S(\boldsymbol{Y}) .
$$

At this order of approximation, it is found that $D_{m}^{c, s}(\epsilon)=0$ for $m \geqslant 2$ and that $\epsilon J$ becomes negligible in (B 17). Finally, from (B 16) and (B 18) one thus arrives at the far-field behaviour (3.31).

\section{Appendix C. Evaluation of $A, B$ and $S$}

This Appendix briefly details the calculation of the coefficients $A$ and $B$, defined by (3.33) and (3.34) respectively and the constant $S$ arising in (4.26). By virtue of (3.29), this task consists of evaluating the real quantities $T^{c}$ and $T^{s}$ such that

$$
\frac{T^{c}+\mathrm{i} T^{s}}{\tilde{c}}=\operatorname{pf} \int_{\mathbb{R}^{2}} \frac{\left[\alpha_{1}^{c}(0) \cos \varphi+\mathrm{i} \alpha_{1}^{s}(0) \sin \varphi\right] \exp (\boldsymbol{W} \cdot \boldsymbol{Y}) K_{1}(\tilde{c}|\boldsymbol{Y}|)}{2 \pi|\boldsymbol{Y}|} \mathrm{d} S(\boldsymbol{Y}) .
$$

Setting $t=|\boldsymbol{Y}|$ and introducing $\Lambda$ such that $\boldsymbol{W} / W=\cos \Lambda \boldsymbol{e}_{x}+\sin \Lambda \boldsymbol{e}_{y}$, the use of the key relation (Abramowitz \& Stegun 1965, 9.6.34)

$$
\exp [W t \cos (\Lambda-\varphi)]=I_{0}(W t)+2 \sum_{m \geqslant 1} I_{m}(W t) \cos [m(\Lambda-\varphi)]
$$

easily yields

$$
\begin{array}{ll}
2 T^{c}=\alpha_{1}^{c}(0)\left[k_{0}+k_{2} \cos 2 \Lambda\right]+\alpha_{1}^{s}(0) k_{2} \sin 2 \Lambda, & \frac{k_{0}}{\tilde{c}}=\mathrm{pf} \int_{0}^{\infty} K_{1}(\tilde{c} t) I_{0}(W t) \mathrm{d} t, \\
2 T^{s}=\alpha_{1}^{c}(0) k_{2} \sin 2 \Lambda+\alpha_{1}^{s}(0)\left[k_{0}-k_{2} \cos 2 \Lambda\right], & \frac{k_{2}}{\tilde{c}}=\mathrm{pf} \int_{0}^{\infty} K_{1}(\tilde{c} t) I_{2}(W t) \mathrm{d} t .
\end{array}
$$


Finally, each integral $k_{0}$ or $k_{2}$ is obtained by using the identity $I_{2}(u)=I_{0}(u)-$ $2 I_{1}(u) / u$ and, for $l \in\{0,1\}$, the relations (Abramowitz \& Stegun 1965, 9.6.10 and Gradshteyn \& Ryzhik 1965, p. 684)

$I_{l}(u)=\left(\frac{u}{2}\right)^{l} \sum_{k=1}^{\infty} \frac{(u / 2)^{2 k}}{k !(k+l) !}, \quad \int_{0}^{\infty} x^{2 k} K_{1}(W x) \mathrm{d} x=\frac{(k-1) ! k !}{2 W}\left(\frac{2}{W}\right)^{2 k}, \quad k \geqslant 1$.

If $\gamma$ denotes Euler's constant, the results are

$$
\begin{aligned}
& k_{0}=-\left\{\gamma+\log \left[\frac{\tilde{c}}{2}\right]+\frac{1}{2} \log \left[1-\left(\frac{W}{\tilde{c}}\right)^{2}\right]\right\}, \\
& k_{2}=-\frac{1}{2}\left\{1+\left(\frac{\tilde{c}}{W}\right)^{2} \log \left[1-\left(\frac{W}{\tilde{c}}\right)^{2}\right]\right\}
\end{aligned}
$$

and we immediately deduce (3.37)-(3.38). Finally, some elementary algebra also provides

$$
S=\log \epsilon+2 \gamma-1+2 \log d+\log 2+T_{2}-\frac{S_{2} T_{1}}{S_{1}}
$$

\section{REFERENCES}

Abramowitz, M. \& Stegun, I. A. 1968 Handbook of Mathematical Functions. Dover.

Badr, H. M., Coutanceau, M., Dennis, S. C. R. \& Menard, C. 1990 Unsteady flow past a rotating circular cylinder at Reynolds numbers $10^{3}$ and $10^{4}$. J. Fluid Mech. 220, 459-484.

DorrepaAl, J. M., O’Neill, M. E. \& Ranger, K. B. 1984 Two dimensional Stokes flow with cylinders and line singularities. Mathematika 31, 65-75.

Elliott, L., Ingham, D. B. \& Ei Bashir, T. B. A. 1995 Stokes flow past two circular cylinders using a boundary element method. Computers Fluids 24, 787-798.

Erdélyi, A., Magnus, W., Oberhettinger, F. \& Tricomi, G. 1955 Higher Transcendental Functions, vol.III, chap.XVIII. McGraw-Hill.

FujIKaWA, H. 1956 The forces acting on two circular cylinders of arbitrary radii placed in a uniform stream at low values of Reynolds numbers. J. Phys. Soc. Japan 11, 690-701.

FujIKAWA, H. 1957 Expansion formulae for the forces acting on two equal circular cylinders placed in a uniform stream at low values of Reynolds number. J. Phys. Soc. Japan 12, 423-430.

Gradshteyn, I. S. \& RyzhiK, J. M. 1965 Table of Integrals Series and Product. Academic.

Hadamard, J. 1932 Lecture on Cauchy's Problem in Linear Differential Equations. Dover.

Jeffery, G. B. 1920 Plane stress and Plane strain in bipolar cordinates. Phil. Trans. R. Soc. Lond. A 221, 265-293.

Jeffery, G. B. 1922 The rotation of two circular cylinders in a viscous fluid. Proc. R. Soc. Lond. A 101, 169-174.

KAPLUN, S. 1957 Low Reynolds number flow past a circular cylinder. J. Math. Mech. 6, 595-603.

Kaplun, S. \& Lagerstrom, P. A. 1957 Asymptotic expansions of Navier-Stokes solutions for small Reynolds numbers. J. Math. Mech. 6, 585-593.

KIDA, T. 1991 Matched asymptotic expansion method to integral formulations of wing theories. RIMS Kokyuroku 475, 202-219.

KidA, T. \& TAKE, T. 1995 Singular perturbation of integral equations on wing theories. Proc. 6th Asian Congress of Fluid Mechanics, Singapore (ed. Y. T. Chew \& C. P. Tso), vol. 1, pp. 165-168.

KidA, T. \& UEDA, Y. 2002 Low Reynolds number flow around an impulsively started rotating circular cylinder (Asymptotic approach to a steady flow) Trans. JSME B 68, (6666), 375-383.

Kuwabara, S. 1957 The forces experienced by two circular cylinders in a uniform flow at small Reynolds numbers. J. Phys. Soc. Japan 12, 291-299.

LeE, S. H. \& LeAL, L. G. 1986 Low-Reynolds-number flow past cylindrical bodies of arbitrary cross-sectional shape. J. Fluid Mech. 164, 401-427. 
NAKANishi, M. \& KIDA, T. 1999 Unsteady low Reynolds number flow past two rotating circular cylinders by a vortex method. Proc. 3rd ASME/JSME Joint Fluids Engr. Conf. FEDSM 99-6816.

NAKanishi, M. Kida, T. \& NaKaJima, T. 1997 Asymptotic solutions for two-dimensional low Reynolds number flow around an impulsively started circular cylinder. J. Fluid Mech. 334, $31-59$.

Proudman, I. \& Pearson, J. R. A. 1957 Expansions at small Reynolds numbers for the flow past a sphere and a circular cylinder. J. Fluid Mech. 2, 237-262.

Sellier, A. 1994 Asymptotic expansion of a class of integrals. Proc. R. Soc. Lond. A 445, 693-710.

Sellier, A. 1997 Hadamard's finite part concept in dimension $n \geqslant 2$ : definition and change of variables. Math. Proc. Camb. Phil. Soc. A 122, 131-148.

Shintani, K., Umemura, A. \& Takano, A 1983 Low-Reynolds-number flow past an elliptic cylinder, J. Fluid Mech. 136, 277-289.

Sмith, S. H. 1991 The rotation of two circular cylinders in a viscous fluid. Mathematika 38, 63-66.

Stokes, G. G. 1850 On the effect of the internal friction of fluids on the motion of pendulums. Part I Analytical investigation. Section III Solution of the equations in the case of an infinite cylinder oscillating in an unlimited mass of fluid, in a direction perpendicular to its axis. Reprinted in Mathematical and Physical Papers, Vol III. Cambridge University Press (1901).

TомотікA, S. \& Aor, T. 1950 The steady flow of viscous fluid past a sphere and circular cylinder at small Reynolds numbers. Q. J. Mech. Appl. Maths 3, 140-159.

TомотікA, S. \& AoI, T. 1951 An expansion formula for the drag on a circular cyinder moving through a viscous fluid at small Reynolds numbers. Q. J. Mech. Appl. Maths 4, 401-406.

Ueda, Y., Kida, T., Nakanishi, M. \& Sellier, A. 2001 Low-Reynolds-number flow around an impulsively started rotating and translating circular cylinder. Trans. JSME B 67 (659), 16261633.

Umemura, A. 1982 Matched-asymptotic analysis of low-Reynolds-number flow past two equal circular cylinders. J. Fluid Mech. 121, 345-363.

Van Dyke, M. 1975 Perturbation Methods in Fluid Mechanics. Parabolic.

Watson, E. J. 1995 The rotation of two circular cylinders in a viscous fluid. Mathematika 42, $105-126$.

Watson, E. J. 1996 Slow viscous flow past two rotating cylinders. Q. J. Mech. Appl. Maths 49, $195-215$.

Yano, H. \& Kieda, A. 1980 An approximate method for solving two-dimensional low-Reynoldsnumber flow past arbitrary cylindrical bodies. J. Fluid Mech. 97, 157-179. 